\title{
On the micro-PIV accuracy and reliability utilizing non-Gaussian particle images
}

\author{
Sebastian Blahout ${ }^{1}\left[\right.$ D $\cdot$ Simon R. Reinecke ${ }^{2} \cdot$ Harald Kruggel-Emden $^{2} \cdot$ Jeanette Hussong ${ }^{1}$
}

Received: 7 August 2020 / Revised: 6 August 2021 / Accepted: 6 August 2021 / Published online: 28 August 2021

(c) The Author(s) 2021

\begin{abstract}
Optical investigations of the dynamics of concentrated suspensions, such as in blood flows (Fitzgibbon et al. in Biophys $\mathbf{J}$ 108(10):2601-2608, 2015. http://doi/org/10.1016/j.bpj.2015.04.013) or slurry flows (Li et al. in Ocean Eng 163(October 2017):691-705, 2018. http://doi/org/10.1016/j.oceaneng.2018.06.046), are challenging due to reduced optical accessibility. Furthermore, the suspension particle image size can strongly deviate from the optimal particle image size for PIV measurements. Optical accessibility can be achieved by refractive index matching of surface labelled suspension particles. This results in particle images that are transparent in the particle image centre, but fluoresce at the particle image rim, resulting in ring-shaped particle images. In the present study, the influence of the particle image size on the cross-correlation result of such ring-shaped particle images is compared with Gaussian and plateau-shaped particle images. Particles of Gaussian image shape result from fully labelled particles with small image diameters and are commonly used in PIV measurements. Such particles are also utilized for the determination of the continuous phase velocities in the experimental part of the present study. With increasing image diameter, fully labelled particles are observed to assume plateau-shaped particle images. Monte Carlo simulations of synthetically generated images show that ring-shaped particle images have a superior behaviour, i.e. they assume a reduced displacement estimation error for noisy as well as for noise-free image data, compared to Gaussian and plateau-shaped particle images. This is also true for large particle image diameters when particle images are intersected at interrogation window borders or when different values of nonzero particle image displacements are considered. The detectability is similar for all three particle image shapes as long as particles do not intersect with the interrogation window border. Interestingly, for intersected particles of large image diameter, ring-shaped particle images show a slightly improved detectability compared to particle images of Gaussian and plateau shape. Furthermore, the detectability is insensitive against a nonzero particle image displacement. The usage of refractive index matched, ring-shaped particle images results in a good optical accessibility of the suspension. This allows to perform simultaneous cross-correlation evaluations on large ringshaped particle images and fluid tracers with Gaussian particle images that are two orders of magnitude smaller compared to suspension particle images. Velocity measurements are taken on a suspension containing $5 \mathrm{vol} \%$ surface labelled, refractive index matched $60 \mu \mathrm{m}$ polymethylmethacrylate (PMMA) particles. Simultaneously, $\mu$ PIV measurements of the carrier liquid flow are performed utilizing $1.19 \mu \mathrm{m}$ fluorescent polystyrene (PS) particles. Measurement results reveal a parabolic shape of the velocity profiles of both phases with a mean slip velocity of $7.4 \%$ at the position of maximum streamwise velocity in a $580 \mu \mathrm{m}$ high trapezoidal channel. An error analysis confirms the presence of these slip velocities within a $68.5 \%$ confidence interval. A measurement uncertainty in the order of magnitude of $\mathcal{O}\left(10^{-1} \mathrm{px}\right)$ is reached for both fluid tracers and suspension particles. Overall, the present study demonstrates theoretically and experimentally that the usage of suspension particles with ring-shaped images is superior compared to Gaussian and plateau-shaped particle images of the same size. Additionally, the present study demonstrates that the usage of ring-shaped particle images allows to investigate suspension bulk dynamics by
\end{abstract}

Sebastian Blahout

blahout@sla.tu-darmstadt.de

1 Institute for Fluid Mechanics and Aerodynamics, Technical

University of Darmstadt, 64287 Darmstadt, Germany

2 Chair of Mechanical Process Engineering and Solids

Processing, Technische Universität Berlin, 10587 Berlin,

Germany 
measuring velocity fields of both the suspended and the continuous phase simultaneously and with an overall uncertainty that is in the same order of magnitude as for standard $\mu$ PIV measurements.

\section{Graphic abstract}

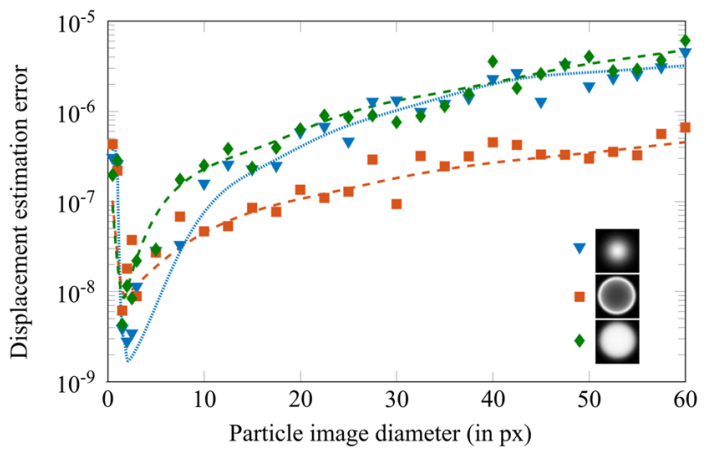

\section{List of symbols}

APTV Astigmatism PTV

CCD Charge-coupled device

$D \quad$ Detectability

$D_{p} \quad$ Particle diameter

$D_{P I} \quad$ Particle image diameter

$d t \quad$ Inter-framing time

$d z \quad$ Spatial distance in $z$ direction

$\Delta d \quad$ Specified particle image displacement

$\delta d \quad$ Calculated particle image displacement

$F_{I} \quad$ In-plane loss-of-pairs factor

$F_{O} \quad$ Out-of-plane loss-of-pairs factor

$h \quad$ Microchannel height

$I\left(\boldsymbol{X}_{i}\right) \quad$ Single exposed image

$I(x) \quad$ Gaussian density function

I Intensity distribution

$K_{5} \quad$ Amount of particle images intersected at interrogation window borders

$l \quad$ Interrogation window edge length

LDV Laser Doppler velocimetry

LIF Laser-induced fluorescence

LL Lower limit

M Magnification

NA Numerical aperture

$N_{I} \quad$ Number of particle images

$n_{D} \quad$ Refractive index at the sodium D-line

$N_{I W} \quad$ Number of interrogation windows

$N_{p p p} \quad$ Particle image density

Nd:YAG Neodymium-doped yttrium aluminium garnet

OPF One-phase flow

PIV Particle Image Velocimetry

PMMA Polymethylmethacrylate

PS Polystyrene

PTV Particle Tracking Velocimetry

$R(s) \quad$ Cross-correlation function

$R_{0} \quad$ Maximum value of highest correlation peak
$R_{C}(s) \quad$ Mean intensity cross-correlation function

$R_{D}(s) \quad$ Particle image displacement cross-correlation function

$R_{F}(s) \quad$ Fluctuating noise cross-correlation function

$R_{ \pm 1} \quad$ Correlation values next to $R_{0}$

$R_{P I} \quad$ Particle image radius

$R_{b} \quad$ Bulk Reynolds number

RMSE Root mean square error

$\boldsymbol{s} \quad$ Separation vector

St Stokes number

SCL Suspension carrier liquid

SP Suspension particles

$\Delta t \quad$ Time interval

$t_{0} \quad$ Reference time

$U \quad$ Uncertainty

$u \quad$ Component of the velocity vector in stream-

wise direction

$u_{b} \quad$ Bulk fluid velocity

UL Upper limit

$v \quad$ Component of the velocity vector in spanwise direction

$W_{1} \quad$ Interrogation area

$\boldsymbol{X}_{i} \quad$ Particle image centre positions

$y \quad$ Coordinate in spanwise direction

$z \quad$ Coordinate in out-of-plane direction

$\delta_{D o C} \quad$ Depth of correlation

$\epsilon_{1}, \epsilon_{2} \quad$ Error contributions to the displacement estimation error

$\hat{\epsilon}_{G} \quad$ Gaussian peak fit estimator

$\mu \quad$ Mean of Gaussian density function

$v \quad$ Kinematic viscosity

$\phi \quad$ Volumetric particle concentration

$\rho \quad$ Density

$\sigma^{2} \quad$ Variance of Gaussian density function

$\operatorname{var}\left\{\hat{\epsilon}_{G}\right\} \quad$ Displacement estimation error 


\section{Introduction}

Particle Image Velocimetry (PIV) is an established method for non-invasive flow measurements. If optical accessibility is granted, it can be applied to any type of particle seeded flow to trace the fluid motion, provided that particles behave as ideal fluid tracers. Usually in PIV measurements, tracer particles are chosen such that they follow the liquid-phase slip-free, i.e. Stokes number $S t \ll 1$. This guarantees that measured tracer particle velocities are representative for the liquid-phase velocity field. However, if the Stokes number or the particle size compared to the characteristic channel dimensions is large, a significant slip velocity between suspended particles and the carrier fluid may occur. Thus, the particle velocities are not representative for the liquid-phase flow field. Instead, particle velocities can be used to measure the bulk dynamics of particles themselves. In the experimental section of the present study (Sect. 5), we demonstrate that the simultaneous usage of both small tracer particles and large suspension particles allows to measure liquid-phase velocities as well as suspension particle velocities from the same set of recordings, i.e. at the same time. PIV is commonly applied to situations of high particle seeding density. It is suitable to measure transient flow fields, also in combination with other optical measurement techniques (Skarman et al. 1996; Funatani et al. 2004; Kordel et al. 2016). In classical PIV applications, fluid tracers should assume a small image diameter of only a few pixels (Willert 1996; Westerweel 1997). Without astigmatism effects, their intensity profile will be typically Gaussian (Adrian 1991; Willert and Gharib 1991). The accuracy and reliability of PIV measurements for Gaussian particle images is a function of various parameters, such as the amount of particles per interrogation window, the particle image density, the particle image diameter, the signal-to-noise ratio, out-of-plane as well as in-plane loss-of-pairs and velocity gradients (Adrian 1991; Willert 1996; Westerweel 1997).

A measure for the accuracy of a cross-correlation result is the displacement estimation error. It is known to minimize for particle image diameters of $D_{P I}=2-3 \mathrm{px}$, while it grows for larger diameters due to random errors (Westerweel 1997). While PIV is typically used to quantify flow fields, it is applicable to any kind of displacement field as long as the particle image shape and particle group formation do not change significantly between correlated frames.

A measure for the reliability of PIV measurements is the detectability, which is the ratio of the highest to the second highest correlation peak. It can be understood as the probability that the highest correlation peak corresponds to the real particle image displacement (Adrian 1991). Detailed information regarding the detectability and the displacement estimation error is given in Sect. 2.
Due to a reduced optical accessibility of suspension flows, PIV measurements of multiphase flows were often limited to dilute suspensions (Koutsiaris et al. 1999) or bubble flows of low gas volume fractions (Lindken and Merzkirch 2002). For optical investigations of suspension flows with higher volume fractions, a refractive index matching of the liquid to the solid phase can be done to increase the optical accessibility (Wiederseiner et al. 2011; Blanc et al. 2013). The concept of refractive index matching can be applied to every liquid-solid combination with a transparent liquid and solid phase. Solely difficulties in handling of highly flammable or toxic liquids may restrict the choice of materials (Hassan and Dominguez-Ontiveros 2008; Wiederseiner et al. 2011). Hence, this concept was already successfully applied for Particle Tracking Velocimetry (PTV) (Wang et al. 2008), laser Doppler velocimetry (LDV) (Haam et al. 2000), laser-induced fluorescence (LIF) (Chen et al. 2005) and Astigmatism Particle Tracking Velocimetry (APTV) (Brockmann et al. 2020; Brockmann and Hussong 2021) measurements in multiphase flows. An advantage of the PIV measurement technique compared to other optical, non-invasive measurement techniques like the APTV approach is that no sophisticated calibration technique is required and all suspension particles can be labelled and, therefore, contribute to the measurement result. In the present study, we apply refractive index matching of a ternary carrier liquid to suspension particles to gain optical access. This mixture is used as a basis for synthetic particle image generation (see Sect. 3) as well as for suspension flow measurements (see Sect. 5.)

To assess the suitability of cross-correlation-based measurement techniques like PIV for measurements in dense suspensions, we evaluate the displacement estimation error and the detectability of zero-displaced Gaussian, ring- and plateau-shaped particle images of different particle image diameters by means of Monte Carlo simulations that are based on synthetically generated particle images (see Sects. 4.1 and 4.2). These show that the accuracy and reliability of particles with large image diameter strongly depends on the particle image shape. While a Gaussian intensity distribution is characteristic for small particle image diameters (Willert and Gharib 1991), particles assume rather a plateau-shaped intensity distribution for large image diameters (see also Sect. 3). To separate the size effect from the shape effect for PIV measurements, we study the behaviour of both Gaussian and plateau-shaped particle images over the whole investigated image size regime. Ring-shaped intensity profiles may emerge for refractive index matched particles with fluorescent surface labelling. This situation may be encountered for optical investigations of dense particle laden flows but also in situations where particles naturally contain mainly carrier liquid such as hydrogel particles (Byron and Variano 2013) or cells (Lima et al. 2006). 
For small particle images, an in-plane loss-of-pairs refers to particles that are located only in one interrogation window of the first or second frame (Keane and Adrian 1992). However, when considering large particle image sizes, the contribution of intersected particle images may become relevant, too. This effect is investigated for Gaussian, ring- and plateau-shaped particle images in Sect. 4.3.

In real measurement situations, particle images are displaced between two consecutive frames. The influence of nonzero particle image displacements on the estimation error, the detectability and the deviation of the resulting displacement vector is investigated based on Monte Carlo simulations in Sect. 4.4.

To understand the dynamics of suspended particles, the relative motion between particles and surrounding fluid is evaluated. Relative motion may, for example, occur when the particle diameter is in the order of magnitude of the characteristic channel dimensions and, therefore, is much larger than the diameter of tracer particles commonly used in PIV measurements. To demonstrate the suitability of the utilized ring-shaped particle images to measure such suspension dynamics, micro-Particle Image Velocimetry ( $\mu$ PIV) measurements are performed (see Sect. 5). As, additionally, small tracer particles are suspended to the flow, a simultaneous evaluation of the velocity profiles of both the continuous and the particulate phase is possible and slip velocities can be calculated.

A flow chart of the structure of the theoretical and the experimental part of the present study is given in Fig. 1.

\section{Theoretical background}

This section deals with the mathematical description of the cross-correlation of two images. The choice of a suitable peak fit estimator is discussed for the determination of displacement vectors (Sect. 2.1). Afterwards, the concept of detectability and displacement estimation error are introduced (Sect. 2.2).

\subsection{Cross-correlation and peak fit estimator}

Two single exposed images $I_{1}\left(\boldsymbol{X}_{1}\right)$ and $I_{2}\left(\boldsymbol{X}_{2}\right)$ recorded at times $t_{0}$ and $t_{0}+\Delta t$ are considered, where $\boldsymbol{X}_{i}$ generally contains the centre positions of all particle images in the respective image. If particle images are displaced during the time interval $\Delta t$ by a separation vector $s$, then it follows that $X_{2}=X_{1}+s$ and the cross-correlation function $R(s)$ of the two images $I_{1}\left(\boldsymbol{X}_{1}\right)$ and $I_{2}\left(\boldsymbol{X}_{2}\right)$ can be defined as (Keane and Adrian 1992):

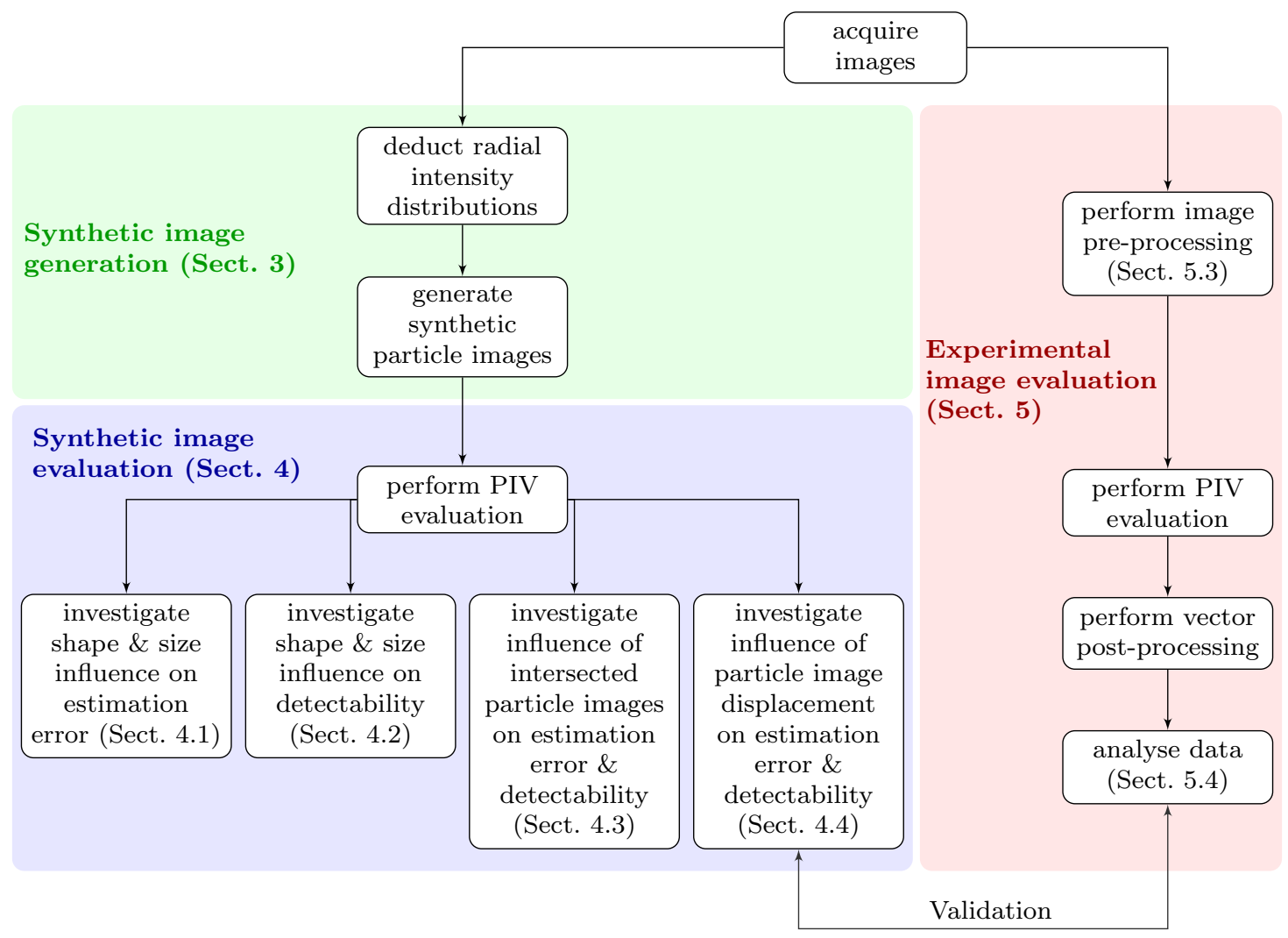

Fig. 1 Overall study flow chart 
$R(\boldsymbol{s})=\int_{W_{1}} I_{1}\left(\boldsymbol{X}_{1}\right) I_{2}\left(\boldsymbol{X}_{1}+\boldsymbol{s}\right) d \boldsymbol{X}$

Here, $W_{1}$ denotes the interrogation area. The separation vector $\boldsymbol{s}$ can be also interpreted as the vector by which the second image $I_{2}\left(\boldsymbol{X}_{2}\right)$ has to be shifted to match the particle positions of the first image, i.e. $\boldsymbol{s}=-\left(\boldsymbol{X}_{2}-\boldsymbol{X}_{1}\right)=-\Delta \boldsymbol{X}_{P}$. According to Keane and Adrian (1992), the estimator for the cross-correlation can be decomposed into three characteristic parts for single exposed double-frame images:

$R(s)=R_{C}(s)+R_{D}(s)+R_{F}(s)$

$R_{C}(s)$ and $R_{F}(s)$ contain the convolution of the mean intensities and the fluctuating noise of $I_{1}(\boldsymbol{X})$ and $I_{2}(\boldsymbol{X})$, respectively. $R_{D}(s)$ results from the relative displacement of particle images between $I_{1}(\boldsymbol{X})$ and $I_{2}(\boldsymbol{X})$ and is hence on referred to as displacement correlation peak. As shown by Willert and Gharib (1991), $R_{D}(s)$ shows a Gaussian peak shape for particle images with a Gaussian intensity distribution. Therefore, a Gaussian peak fit estimator $\hat{\epsilon}_{G}$ can be used to interpolate the centre position of $R_{D}(s)$ from discrete correlation values. With this, peak-locking effects can be reduced and the detection accuracy of the centre position of the displacement correlation peak can be extended to sub-pixel range (Willert and Gharib 1991). The Gaussian peak fit estimator reads (Westerweel 1997):

$\hat{\epsilon}_{G}=\frac{\ln \left(R_{-1}\right)-\ln \left(R_{+1}\right)}{2\left[\ln \left(R_{-1}\right)+\ln \left(R_{+1}\right)-2 \ln \left(R_{0}\right)\right]}$,

with $R_{0}=R_{D}\left(\Delta \boldsymbol{X}_{P}\right)$ and $R_{ \pm 1}=R_{D}\left(\Delta \boldsymbol{X}_{P} \pm 1\right)$. For ringshaped particle images, the corresponding correlation peak values can be approximated by a Gaussian function, as well. Thus, the peak fit estimator described in (3) is used for the investigation of the correlation results based on ring-shaped particle images in the following.

\subsection{Detectability and displacement estimation error}

The quality of PIV measurements or more precisely, the accuracy with which the displacement correlation peak position is determined and the probability that the position of a displacement correlation peak is equivalent to the real particle image displacement, can be evaluated through the displacement estimation error and the detectability. Thus, they are a measure to describe the accuracy and the reliability of a cross-correlation result.

The detectability $D$ of a displacement correlation peak can be interpreted as the probability that a displacement correlation peak is correctly identified as valid and, therefore, corresponds to the real particle image displacement. It is defined as the ratio of the correlation peak values of the highest and the second highest correlation peak of a correlation function $R(s)$ (Coupland and Pickering 1988; Keane and Adrian 1990; Adrian and Westerweel 2011):

$D=\frac{R(s)_{\max , 1}}{R(s)_{\max , 2}}$

The displacement estimation error can be expressed as the variance of its corresponding peak fit estimator. For a Gaussian peak fit estimator, it can be described in a general mathematical form as (Westerweel 1997):

$\operatorname{var}\left\{\hat{\epsilon}_{G}\right\} \approx \sum_{i=-1}^{+1} \sum_{j=-1}^{+1} \frac{\partial \hat{\epsilon}_{G}}{\partial R_{i}} \frac{\partial \hat{\epsilon}_{G}}{\partial R_{j}} \operatorname{cov}\left\{R_{i}, R_{j}\right\}$

In case of a non-fractional displacement, (5) can be expressed as follows:

$\operatorname{var}\left\{\hat{\epsilon}_{G}\right\} \approx \epsilon_{1} \cdot \epsilon_{2}$,

with both terms $\epsilon_{1}$ and $\epsilon_{2}$ representing different properties of the displacement correlation peak. The first term $\epsilon_{1}$ is the squared derivative of the Gaussian peak fit estimator $\hat{\epsilon}_{G}$ given in (3):

$$
\begin{aligned}
\epsilon_{1} & =\left(\frac{\partial \hat{\epsilon}_{G}}{\partial R_{ \pm 1}}\right)^{2} \\
& =\left(\frac{4 \cdot\left[\ln \left(R_{\mp 1}\right)-\ln \left(R_{0}\right)\right]}{R_{ \pm 1}\left[2 \cdot \ln \left(R_{ \pm 1}\right)-4 \ln \left(R_{0}\right)+2 \cdot \ln \left(R_{\mp 1}\right)\right]^{2}}\right)^{2}
\end{aligned}
$$

If the correlation peak flattens out, i.e. $R_{ \pm 1} \rightarrow R_{0}$, the denominator of (7) approaches zero sooner than the nominator and $\epsilon_{1}$, and therefore, also $\operatorname{var}\left\{\hat{\epsilon}_{G}\right\}$ goes to infinity. The second term $\epsilon_{2}$ reads:

$\epsilon_{2}=\left[\operatorname{var}\left\{R_{-1}\right\}+\operatorname{var}\left\{R_{+1}\right\}-2 \operatorname{cov}\left\{R_{-1}, R_{+1}\right\}\right]$

It is a measure of (i) the spread of the normalized correlation peak slope, i.e. its width as well as (ii) the spread of the correlation peak symmetry of an ensemble. For an ensemble with only perfectly symmetric correlation peaks (that is, $R_{+1}=R_{-1}$ ), the second term and (6), respectively, will approach zero for a finite correlation peak width.

\section{Synthetic particle image generation}

In the present study, we investigate the influence of the particle image diameter on the cross-correlation result for different particle image shapes. For this, synthetic particle images are generated with Gaussian, ring- and plateau-shaped intensity profiles. While Gaussian intensity profiles are based on an analytical function, ring- and plateau-shaped profiles are 
taken from fit functions of experimental image data. For this, self-labelled and commercially labelled PMMA particles with a nominal particle diameter of $D_{p}=60 \mu \mathrm{m}$ are suspended in a carrier liquid and recorded under laser illumination. Examples of both ring- and plateau-shaped particle images with $D_{P I} \approx 70 \mathrm{px}$ image diameter are shown in Fig. 2a, d, respectively. A particle image of Gaussian shape is given in Fig. $2 \mathrm{~g}$.

Obviously, self-labelled PMMA particles assume a ringshaped particle image, while commercially labelled particles show a plateau-shaped intensity profile. To achieve ringshaped particle images, first a molecular Rhodamine B dye is solved in distilled water. Afterwards, PMMA particles are suspended in this solution. After half an hour at room temperature, particles are recovered by sedimentation. The remaining solution of water and Rhodamine B is removed. In the end, labelled particles are dried in a heat bath at $70{ }^{\circ} \mathrm{C}$. After this procedure, particles show to have a limited take up of dye, such that only the particle surface is labelled. When the particles are suspended in a refractive index matched liquid consisting of distilled water, glycerine and

(a)

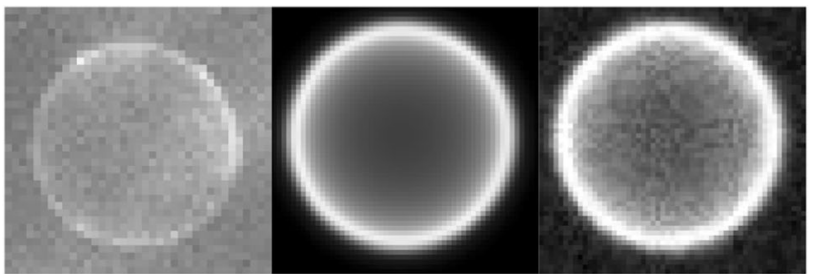

(d)

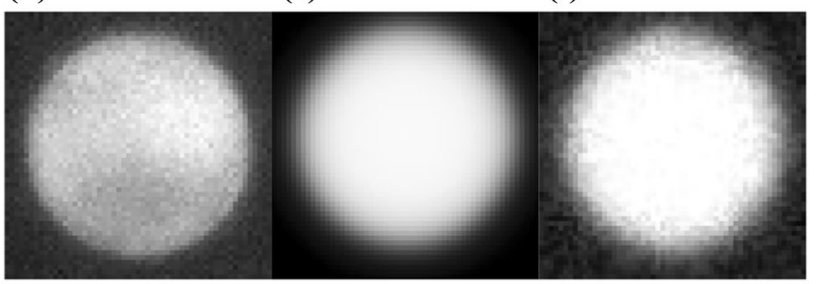

(g)

(h) ammoniumthiocyanate (Bailey and Yoda 2003), they appear transparent with a fluorescent particle rim. In contrast to this, the material of commercially available particles is completely labelled, resulting in a plateau-shaped particle image.

Radial intensity distributions of ring- and plateau-shaped particle images are determined from approximately 500 recorded, individual particle images by fitting a smoothing spline to all intensity distributions. With that, different synthetic particle image diameters are realized for synthetic data generation of ring-shaped and plateau-shaped particle images by scaling the smoothing spline function. To create synthetic Gaussian particle images, the Gaussian density function is used:

$I(x)=\frac{1}{\sqrt{2 \pi \sigma^{2}}} \cdot \exp \left(-0.5 \cdot \frac{(x-\mu)^{2}}{\sigma^{2}}\right)$,

with $\mu=0$. The variance of the Gaussian density function $\sigma^{2}$ is adjusted iteratively, so that the width of the Gaussian density function corresponds to the prescribed particle image diameter. This width is defined to be at the point where the Gaussian curve reaches $0.5 \%$ of its maximum value. Finally, this threshold value is subtracted from the resulting shape function to reach a zero intensity value at $R_{P I} / R_{P I, \max }=1$. The resulting radial intensity distributions $I\left(R_{P I} / R_{P I, \max }\right)$ normalized by the corresponding maximum intensity $I_{\max }$ of all three particle image shapes are shown in Fig. 3.

To create particle images of different discrete pixel sizes, radial intensity distributions are intersected and piecewise averaged intensity values are assigned to the corresponding pixel locations. Synthetic ring- and plateau-shaped particle images of $D_{P I}=60$ px are shown in Fig. 2b, e, respectively. As experimental data are usually affected by image noise, both, synthetic particle images without (see Fig. 2b, e, h) and with $8.5 \%$ image noise (see Fig. 2c, f, i), are analysed and compared in the course of this paper. Image noise is calculated as the ratio of the mean background intensity

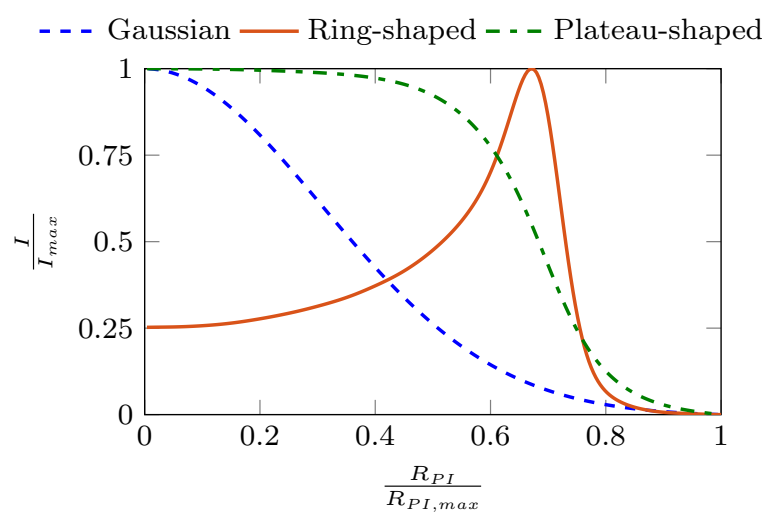

Fig. 3 Radial, fitted intensity distributions of Gaussian, ring- and plateau-shaped particle images 


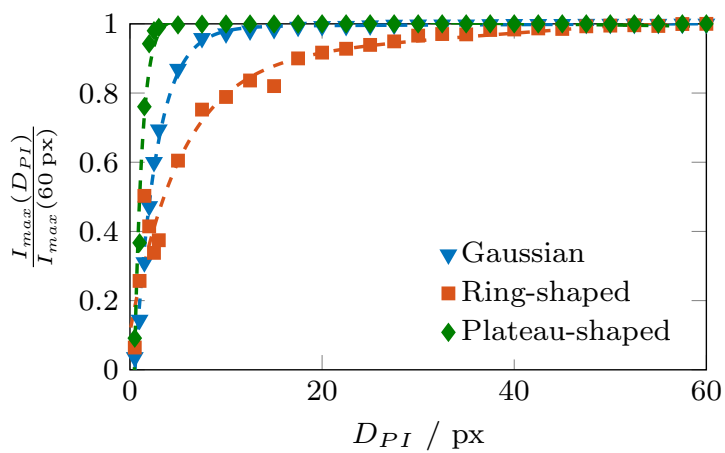

Fig. 4 Maximum intensity values as a function of the particle image diameter for Gaussian, ring- and plateau-shaped particle images. Dashed lines denote fits to the respective data

and the maximum intensity value of images with a particle image diameter of $D_{P I}=60 \mathrm{px}$. Noise levels in the order of magnitude as mentioned above are encountered, even after pre-processing of raw recordings of suspension flows with ring-shaped particle images.

It may be noted that assigning a continuous intensity function (as given in Fig. 3) to discrete pixels leads to a reduction of the maximum particle intensity values for small particle image diameters. Figure 4 shows the intensity maxima as a function of the particle image diameter.

As can be seen, this discretization error becomes prominent for small particle images of a few pixels diameter and is strongest for ring-shaped particle images due to a very sharp intensity peak at $R_{P I} / R_{P I, \max } \approx 2 / 3$, evident in Fig. 3 . However, in real experimental situations where, for example, noise-affected ring-shaped particle images may indeed give a weaker fluorescence signal for identical laser energy input compared to noise-affected plateau-shaped particle images, the cross-correlation result may be significantly affected for particle image diameters of $D_{P I} \leq 10 \mathrm{px}$. Further details are discussed in Sect. 4.

To evaluate the size sensitivity of Gaussian, ring- and plateau-shaped particle images on the cross-correlation result, 500 double-frame images with five randomly distributed particle images in each interrogation window are created synthetically. To investigate solely the influence of the particle image shape on the cross-correlation result by means of the detectability and the displacement estimation error, defocused particle images are not considered, i.e. all particle images are in focus in all synthetically generated images. Furthermore, particle images either assume a zero displacement between consecutive frames or, in case of nonzerodisplaced particle images, are always fully located inside interrogation windows. Due to this, neither an in-plane lossof-pairs nor an out-of-plane loss-of-pairs is present and the corresponding factors with which these effects are taken into account, $F_{I}$ and $F_{O}$, respectively, assume $F_{I}=F_{O}=1$. This results in a constant effective number of particle images of $N_{I} F_{I} F_{O}=N_{I}=5$ for all particle image diameters, which is in agreement with the recommended value for PIV measurements (Keane and Adrian 1992). The displacement estimation error is evaluated based on (6). For all investigations the interrogation window size is chosen to be $256 \times 256 \mathrm{px}$. At first, overlapping particle images as well as particle images intersected at the interrogation window border are suppressed to isolate the effect of the particle image size and shape on the cross-correlation result (see Sects. 4.1 and 4.2). Thus, particle images are also zero-displaced between two consecutive frames.

The influence of zero-displaced particle images that are located on the interrogation window border is investigated separately in Sect. 4.3. For this, sets of double-frame images with one, three and five out of five particle images are placed with their centre point on the interrogation window border. Particle images inside the interrogation window and those on its border are distributed randomly whereas particle overlaps are excluded again. Examples of synthetically generated ring-shaped particle images of $D_{P I}=60 \mathrm{px}$ with zero and three intersected particle images are shown in Fig. 5a, b.

The effect of nonzero particle image displacements on the cross-correlation result is investigated in Sect. 4.4. Overlapping particle images and intersections at the interrogation window borders are excluded.

\section{Results}

To evaluate the influence of the particle image diameter and shape on the displacement estimation error and the detectability, Monte Carlo simulations based on ensembles of 500 double-frame images are performed. For the crosscorrelation of synthetically generated data (see Sect. 3), a
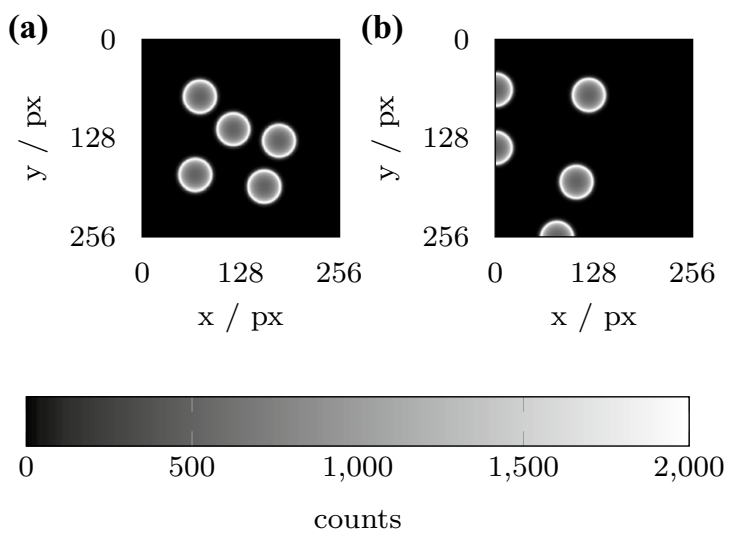

Fig. 5 Synthetic sample images of a five ring-shaped particle images and $\mathbf{b}$ five ring-shaped particle images including three intersected particle images $\left(K_{5}=3\right)$ 
commercial PIV evaluation software (DaVis 8.4, LaVision $\mathrm{GmbH})$ is used.

\subsection{Influence of particle image shape and diameter on the estimation error}

In the present section, the influence of the particle image shape and diameter on the displacement estimation error is described. Excluded is the effect of particle images that are intersected at interrogation window borders, which is discussed in Sect. 4.3.

Figure $6 \mathrm{a}-\mathrm{f}$ shows single cross-correlation results for two particle image sizes of synthetic image data with Gaussian, ring- and plateau-shaped particle images.

Cross-correlation results for particle image diameters of $D_{P I}=5$ px (Fig. 6a, c, e), as well as $D_{P I}=60$ px (Fig. 6b, $\mathrm{d}, \mathrm{f})$ and zero displacement are shown on the left and right hand side, respectively. It is evident that the correlation peak width increases with particle image diameter for all three types of particle image shapes. Furthermore, a comparison (a)

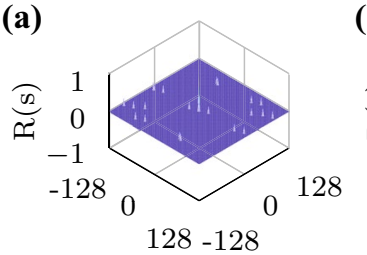

(b)

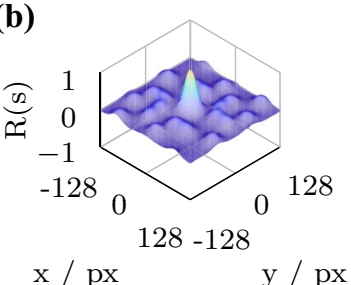

(c)

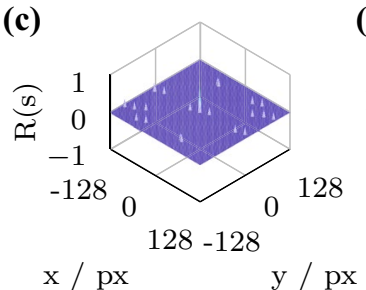

(d)

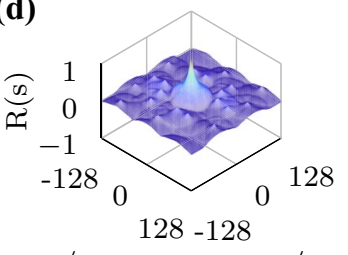

(e)

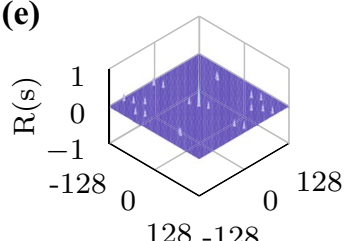
$128-128$ (f)

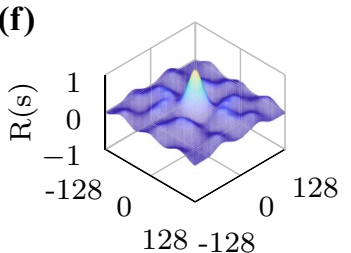

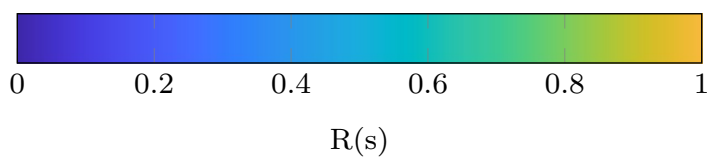

Fig. 6 Correlation maps resulting from the cross-correlation for a zero displacement of synthetically generated images with five a $5 \mathrm{px}$ Gaussian, b 60 px Gaussian, c 5 px ring-shaped, d 60 px ring-shaped, e 5 px plateau-shaped and f 60 px plateau-shaped particle images of displacement correlation peaks close to their maximum values reveals that correlation peaks resulting from ring-shaped particle images are most narrow compared to those of Gaussian and plateau-shaped particle images (see Fig. 7a-c).

The scaling behaviour between particle image shape and displacement estimation error is evident in the correlation peak width [see (6)]. Here, the first term $\epsilon_{1}$ grows with an increasing displacement correlation peak width, while the second term $\epsilon_{2}$ of the displacement estimation error denotes random errors. Thus, in comparison with Gaussian particle images a reduced estimation error is expected for ringshaped particle images while a slightly increased estimation error is expected for plateau-shaped particle images. Figure 8 shows the displacement estimation error in a semilogarithmic scale as a function of the particle image diameter derived from a Monte Carlo simulation for Gaussian, ring- and plateau-shaped particle images.

Every data point results from 500 cross-correlated double frames, each containing five particle images and zero displacement between corresponding frames. All graphs resemble a non-monotonic relationship with a minimum estimation error at $D_{P I} \approx 2-3 \mathrm{px}$ as it is also shown for Gaussian particle images by Westerweel (1997). Obviously, this minimum value corresponds to the optimum particle image diameter for PIV measurements as also given in the common literature (Raffel et al. 2007; Adrian and Westerweel (a)

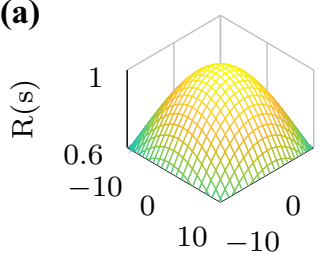

$\mathrm{x} / \mathrm{px}$ (b)

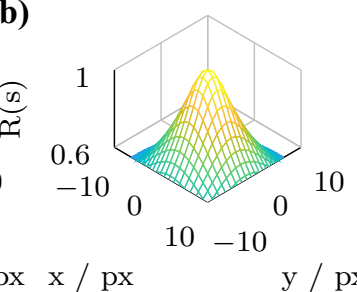

(c)
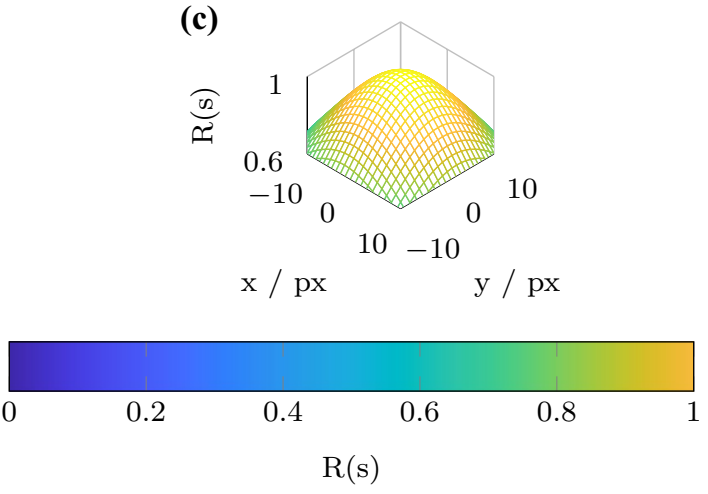

Fig. 7 Zoomed in view of displacement correlation peak tops resulting from the cross-correlation of double-frame images, each containing five particle images with zero displacement between corresponding frames, using a Gaussian, b ring-shaped and c plateau-shaped particle images of $D_{P I}=60 \mathrm{px}$ 


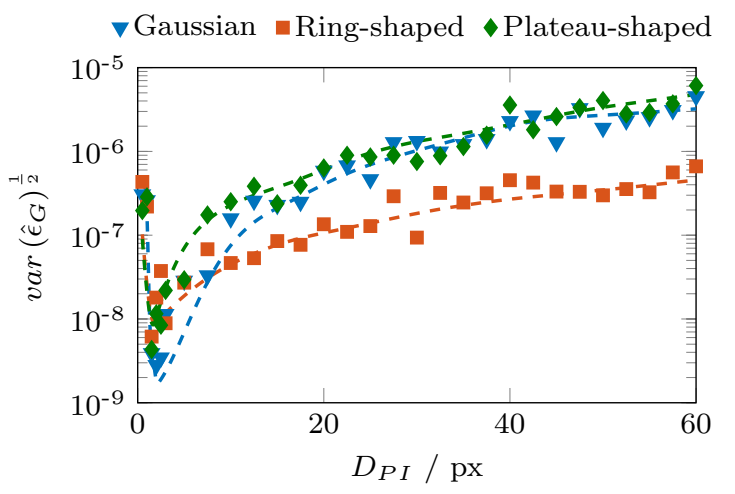

Fig. 8 Estimation error as a function of the particle image diameter (without noise, based on 500 samples per particle image diameter). Dashed lines denote fits to the respective data

2011). For smaller particle image diameters, peak-locking effects occur, which result in an increased estimation error. For larger particle image diameters random errors lead to an increased estimation error (Westerweel 1997). While the estimation error appears to stay in the same order of magnitude for Gaussian and plateau-shaped particle images with growing image diameter, a clear distinction has to be made for ring-shaped particle images for $D_{P I} \gtrsim 10 \mathrm{px}$. Figure 8 shows a reduction of the displacement estimation error of up to one order of magnitude for ring-shaped particle images compared to Gaussian or plateau-shaped particle images (for $D_{P I} \gtrsim 10 \mathrm{px}$ ). This can be understood from the fact that, for a constant particle image diameter, ring-shaped particle images decorrelate faster than Gaussian or plateaushaped particle images, due to their large intensity gradient at the particle image border and a nearly transparent centre region. Thus, the correlation peak width decreases (see also Fig. 6d), resulting in an improved displacement estimation error.

Figure 8 displays results for noise-free data only, a situation that is hardly found in experiments. To validate cross-correlation results of images that are closer to preprocessed experimental measurement data, image noise of $8.5 \%$ is added (for details of the synthetic image generation see Sect. 3) and the data set is analysed as well. The resulting estimation errors are shown in Fig. 9.

A clear difference in displacement estimation error for all three particle image shapes is evident in Fig. 9. However, image noise leads to a strong increase in random errors in the correlation plane (Meinhart et al. 2000). Thus, in comparison with the noise-free data that are shown in Fig. 8, the overall level of displacement estimation error is now increased by approximately four orders of magnitude. This is due to an increase in the correlation peak asymmetry, represented by $\epsilon_{2}$ in (6), i.e. random errors in the correlation plane.

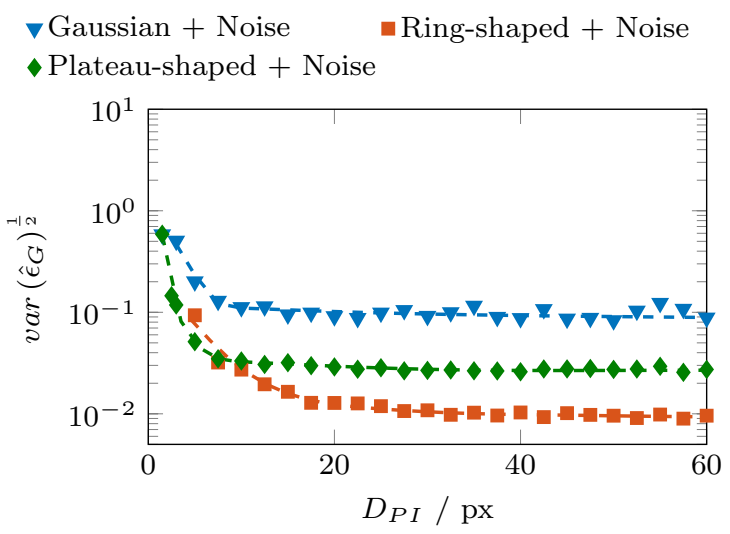

Fig. 9 Estimation error as a function of the particle image diameter (with noise, based on 500 samples per particle image diameter). Dashed lines denote fits to the respective data

Furthermore, it is remarkable to see that noisy Gaussian particle images have a significantly higher estimation error compared to noisy plateau-shaped particle images while the corresponding noise-free particle images lead to very similar displacement estimation errors (see Fig. 8). Figure 10 shows both $\epsilon_{1}$ and $\epsilon_{2}$ as a function of the particle image diameter $D_{P I}$ for noise-free and noisy image data. It should be noted that results of $\epsilon_{1}$ for images with and without noise coincide. Therefore, results of noisy image data are left out in Fig. 10 for a clearer presentation.

As shown in Fig. 10, the increased estimation error of Gaussian particle images results from an increased amount of random errors in the correlation plane. This is obvious, since the values of $\epsilon_{1}$ are similar for Gaussian and plateaushaped particle images with and without noise, but values of $\epsilon_{2}$ differ significantly.

For particle image diameters of $D_{P I}<3 \mathrm{px}$, the particle image diameter is of the same length scale as the image noise, leading to an increased asymmetry of individual

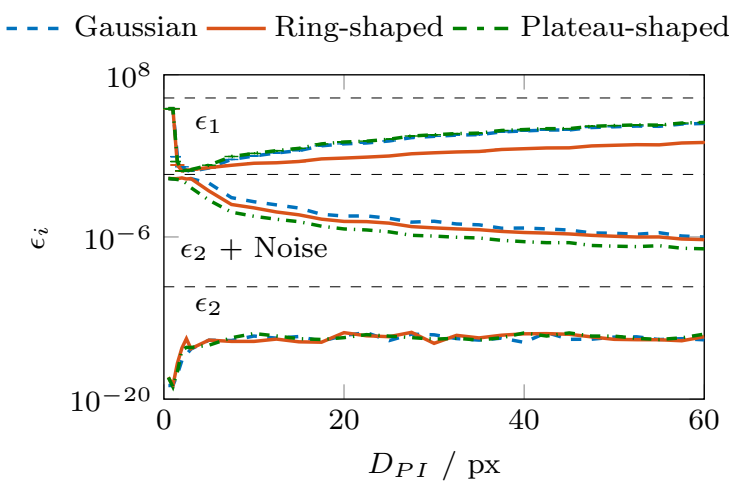

Fig. 10 Factors $\epsilon_{1}$ and $\epsilon_{2}$ of (6) for Gaussian, ring- and plateau-shaped particle images with and without noise. For a clearer presentation, results of $\epsilon_{1}$ for images with noise are not plotted, as they coincide with corresponding results without image noise 
particle image peaks. This induces an increase in random errors represented by $\epsilon_{2}$, as shown in Fig. 10. Here, $\epsilon_{2}$ for images with noise shows increased values for all three particle image shapes, compared to the values of $\epsilon_{2}$ without noise. For larger particle images the random error decreases as the particle image diameter becomes significantly larger than the length scale of the image noise.

\subsection{Influence of the particle image size and shape on the detectability}

Figure 11 shows the detectability $D$ as a function of the particle image diameter for Gaussian, ring- and plateau-shaped particle images without and with image noise of $8.5 \%$.

A general decrease in the detectability with increasing particle image diameter can be associated with a significant increase in the particle image density $N_{p p p}$, i.e. the portion of pixels that is occupied by particle images relative to the total amount of pixels within an interrogation window increases. While the effective number of particle images is constant ( $N_{I}=5$ for all images), the particle image density increases from $N_{p p p} \approx 0.00006$ for $D_{P I}=1$ px to $N_{p p p} \approx 0.22$ for $D_{P I}=60 \mathrm{px}$. Since the particle image density $N_{p p p}$ mainly influences the height of the secondary correlation peak (Scharnowski et al. 2018), an increase in particle image diameter and $N_{p p p}$, respectively, reduces the detectability for a constant $N_{I}$. Furthermore, Fig. 11 shows that the detectability decreases slower for growing ring-shaped particle images compared to those with Gaussian or plateau shape. We assume that this is due to the characteristics of ring-shaped particle images, as slightly shifted ring-shaped particle image groups decorrelate faster than corresponding Gaussian or plateau-shaped particle image groups.
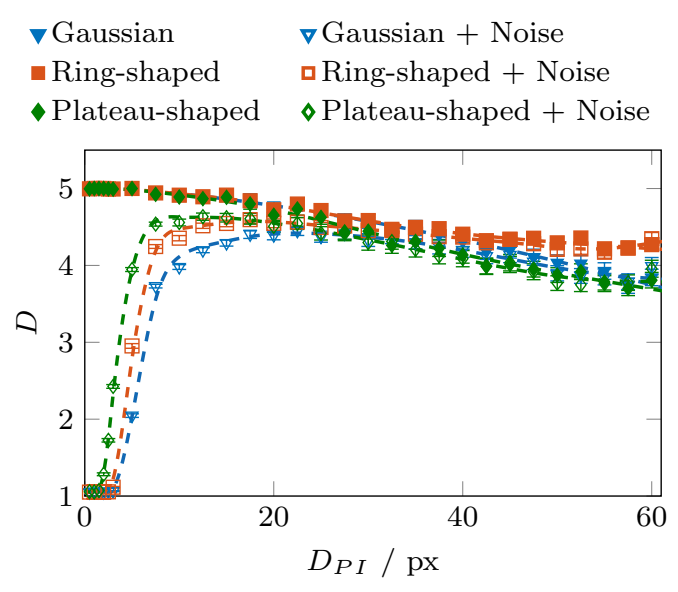

Fig. 11 Detectability with $95 \%$ confidence interval as a function of the particle image diameter averaged over 500 samples per particle image diameter. Dashed lines denote fits to the respective data
Figure 11 also shows the detectability as a function of the particle image diameter for images with noise (as described in Sect. 3). Obviously, image noise affects especially the detectability for small particle image diameters $\left(D_{P I}<10 \mathrm{px}\right)$. This is to be expected, as for small particle images the signal-to-noise ratio is decreased, leading to a lower detectability and thus to an increased probability of erroneous cross-correlations. A reduced signal-to-noise ratio originates from particle image discretization where very narrow particle image intensity peaks are averaged over a full pixel, thereby being reduced in their maximum intensity value (see also Fig. 4). This effect is well known from classical PIV experiments where one strives for particle image diameters of two to three pixels.

\subsection{Influence of intersected particle images on the cross-correlation result}

To study the influence of intersected particle images on the cross-correlation result, three cases are considered for one, three and five out of five particle images located with their centre points on the interrogation window border. These cases with particle images located only to $50 \%$ inside the interrogation window are denoted with $K_{5}=1,3$ and 5, respectively, where the index of $K$ denotes the total amount of particle images inside the corresponding interrogation window. An intersection ratio of $50 \%$ is chosen, because the amount of pixels at which an intensity gradient jump occurs is largest for this situation. Thus, the influence of intersected particle images on the displacement estimation error and the detectability is assumed to be most significant.

Figure 12 shows the displacement estimation error as a function of the particle image diameter for $K_{5}=1,3$ and 5 for Gaussian and ring-shaped particle images. Since the

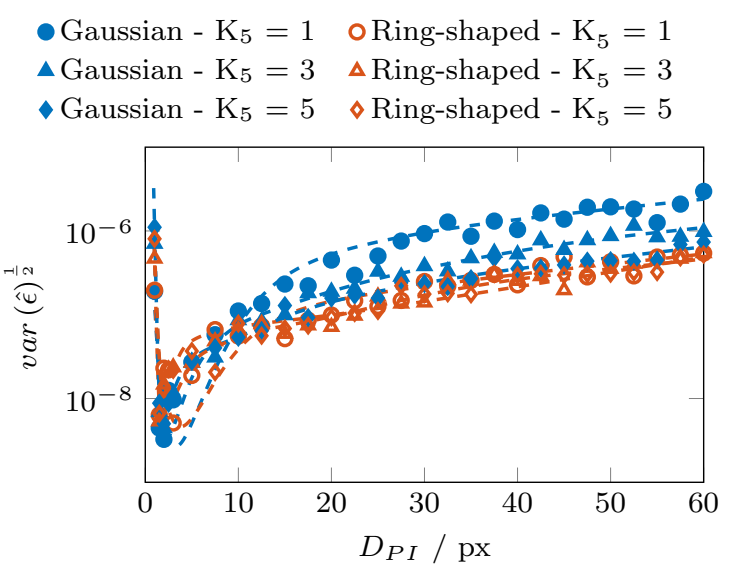

Fig. 12 Estimation error as a function of particle image diameter for one, three and five out of five particle images intersected at the interrogation window border. Dashed lines denote fits to the respective data 
results of plateau-shaped particle images strongly coincide with that of Gaussian particle images, results of plateaushaped particle images are omitted here. It is obvious that the amount of intersected particle images has a stronger influence on the displacement estimation error for Gaussian particle images compared to ring-shaped particle images.

Figure 13 displays the displacement estimation error as a function of $K_{5}$ for Gaussian, ring- and plateau-shaped particle images of $D_{P I}=60 \mathrm{px}$.

The displacement estimation error reduces for intersected Gaussian and plateau-shaped particle images for increasing $K_{5}$-values. This is because intersected particle images deliver a sharp intensity jump at the interrogation window border. Such a sharp intensity jump leads to a reduction in the correlation peak width and hence an improved displacement estimation error. As more particles are intersected, their contribution to the cross-correlation result enhances the aforementioned effect. As already shown in Sect. 4.1, ring-shaped particle images decorrelate faster for slight image shifts due to the relatively small ring width and the transparent inner region. This is also the reason why the effect of intersected particle images on the estimation error is strongly reduced compared to Gaussian and plateau-shaped particle images. Nevertheless, it should be noted that the development of the displacement estimation error may change significantly if particle images within an interrogation window are displaced by values other than zero. Especially if the particle image displacement is smaller than the portion of particle images that is located inside the interrogation window, cross-correlation-based evaluations may lead to systematic errors. To overcome this, apodization window functions as they are described by Eckstein et al. (2008) and Eckstein and Vlachos (2009) may be used. Nevertheless, such apodization window functions have not been applied in the present study.

The influence of intersected particle images at the interrogation window border on the detectability $D$ is illustrated in

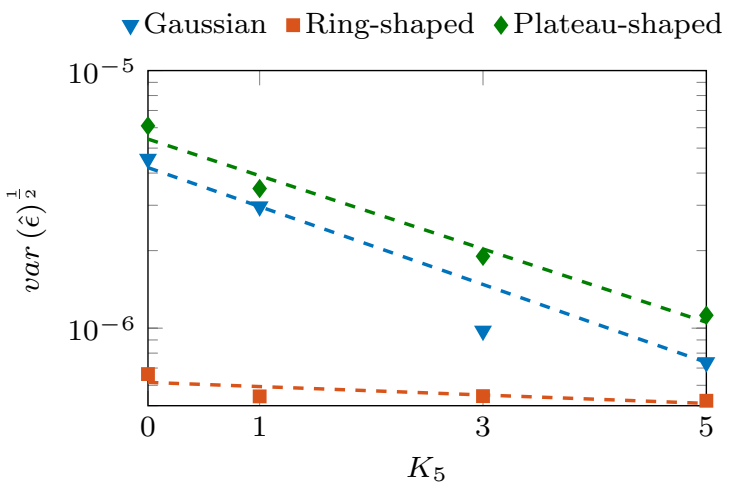

Fig. 13 Displacement estimation error as a function of the number of intersected particle images $K_{5}$ for Gaussian, ring- and plateaushaped particle images of $D_{P I}=60 \mathrm{px}$. Dashed lines denote fits to the respective data
Figs. 14 and 15 . Figure 14 shows how $D$ alters as a function of the particle image diameter $D_{P I}$ for Gaussian and ringshaped particle images with $K_{5}=1,3$ and 5. For a better conciseness, curves for plateau-shaped particle images are omitted as they show no significant differences to those of Gaussian particle images.

Comparing different values of $K_{5}$, a qualitative change in the detectability evolution can be recognised. While a monotonous decrease in the detectability can be observed for $K_{5}=1$ with growing particle image size, it appears to be non-monotonous for $K_{5}=3$ and $K_{5}=5$. This qualitative behaviour is found for all particle image shapes and indicates that different competing effects come into play here. Firstly, a decrease in detectability with increasing particle image size is also observed when no particle images intersect the interrogation window border (see Fig. 11). It originates from the relative increase in secondary correlation peak values

$$
\begin{array}{ll}
- \text { Gaussian }-\mathrm{K}_{5}=1 & \text { oRing-shaped }-\mathrm{K}_{5}=1 \\
\Delta \text { Gaussian - } \mathrm{K}_{5}=3 & \Delta \text { Ring-shaped }-\mathrm{K}_{5}=3 \\
\Delta \text { Gaussian - } \mathrm{K}_{5}=5 & \diamond \text { Ring-shaped - } \mathrm{K}_{5}=5
\end{array}
$$

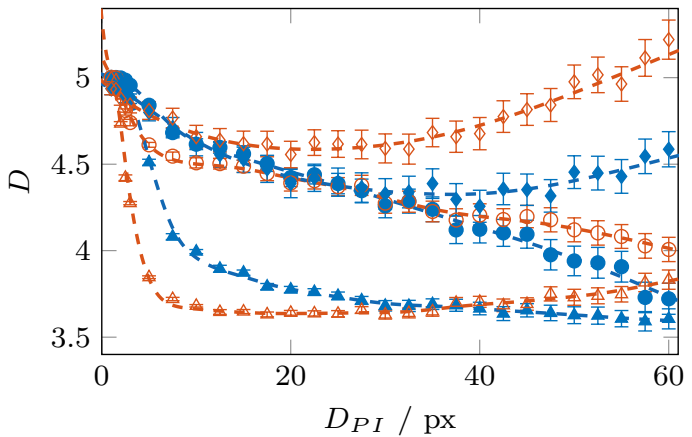

Fig. 14 Detectability with $95 \%$ confidence interval for Gaussian and ring-shaped particle images as a function of the particle image diameter for $K_{5}=1,3$ and 5. Dashed lines denote fits to the respective data

$\nabla$ Gaussian $\square$ Ring-shaped $\diamond$ Plateau-shaped

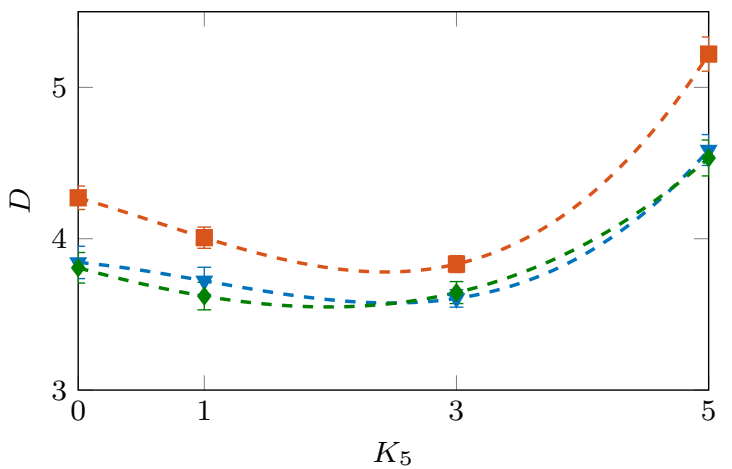

Fig. 15 Detectability with $95 \%$ confidence interval as a function of the number of intersected particle images $K_{5}$ for Gaussian, ring- and plateau-shaped particle images of $D_{P I}=60 \mathrm{px}$. Dashed lines denote fits to the respective data 
that are a result of an increase in the particle image density $N_{p p p}$ for a constant effective number of particle images $N_{I}$. A growing number of intersected particle images is in our case associated with a decreasing number of particle images located inside the interrogation window (as the total number of particle images is chosen to be constant here). Thus, an increasing number of intersected particle images leads to a decreased particle image density $N_{p p p}$, which in turn results in an improved detectability due to a decrease in secondary correlation peak values. However, the overall detectability seems to be lowest for $K_{5}=3$ while it is clearly enhanced for smaller or larger $K_{5}$ values. Therefore, this effect alone does not explain the overall behaviour. We assume that the detectability is decreased as the probability of erroneous correlations resulting from particle images inside the interrogation window correlating with intersected particle images is largest for $K_{5} \approx 1 / 2 \cdot N_{I}$. This is due to a similar number of particle images inside the interrogation window and on the interrogation window border, respectively. Furthermore, the location of particle images at the interrogation window border is strongly restricted. This obviously leads to a relative increase in secondary correlation peak values and hence a reduced detectability. It should be noted that, to overcome a decrease in detectability due to a certain amount of intersected particle images, apodization window functions may be applied.

Figure 15 shows detectability values of Gaussian, ringand plateau-shaped particle images of $D_{P I}=60 \mathrm{px}$ diameter as a function of $K_{5}$.

The detectability shows a non-monotonic behaviour for growing $K_{5}$ values. Furthermore, the detectability of Gaussian and plateau-shaped particle images assumes similar values (see also Fig. 11), while it is slightly increased for ringshaped particle images. It may be noted here that values of $K_{5}=5$ are a hypothetical case. However, for high-resolution studies on dense particle systems such as suspension microflows, large values of $K$ can be expected as $K$ scales with the particle image diameter or, respectively, decreasing interrogation window size.

Figure 16 illustrates the evolution of the ratio of $K$ to the total amount of particle images per interrogation window as a function of the ratio between interrogation window edge length $l$ to particle image diameter $D_{P I}$, for in-plane particle images with simple cubic packing (see inset of Fig. 16).

As can be seen, values in the order of one can be expected for a ratio of interrogation window size and particle image diameter of $l / D_{P I}<5$ - a situation easily encountered in suspension flow studies.

Similar qualitative results in terms of estimation error and detectability as shown in Figs. 13 and 15 are also obtained from experiments. Both the estimation error and detectability are determined for experimental images containing ring- or plateau-shaped particle images of $D_{P I}=60 \mu \mathrm{m}$,

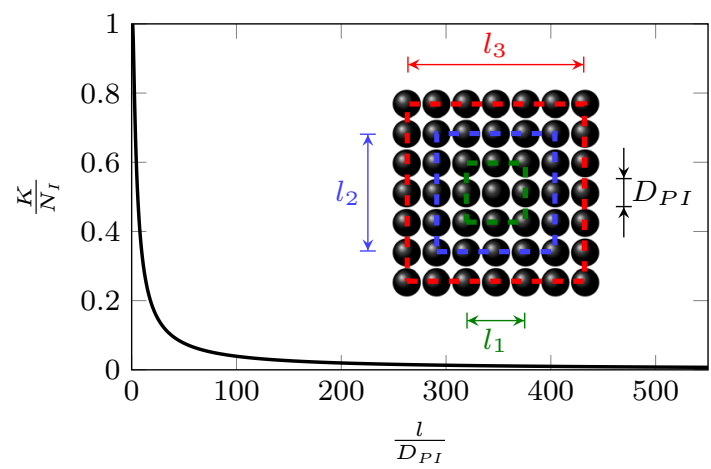

Fig. 16 Development of the ratio of the amount of intersected particle images and the total amount of particle images per interrogation window $K / N_{I}$ as a function of the ratio of the corresponding interrogation window length and the particle image diameter $l / D_{P I}$

respectively. Images are auto-correlated in the commercial software DaVis 8.4 (LaVision $\mathrm{GmbH}$ ) utilizing decreasing interrogation window sizes. The latter results in interrogation window length-to-particle image diameter ratios of $l / D_{P I}=5.3,2.6,1.3,0.7$ and 0.3. Similar to the results obtained from synthetically generated images, also for experimental images the dominant term of the displacement estimation error $\epsilon_{1}$ is one to two orders of magnitude smaller for ring-shaped particle images than for plateau-shaped particle images (see also Figs. 10 and 13). This holds true for all considered values of $l / D_{P I}$.

The detectability of experimental ring-shaped particle images is slightly larger than for experimental plateaushaped particle images for $l / D_{P I}=5.3$. Qualitatively, this is in accordance to the detectability results obtained from synthetic data for $l / D_{P I}=4.3$ (see also Fig. 15). For smaller values of $l / D_{P I}$, the variation of the detectability of experimental ring-shaped particle images increases, such that similar detectabilities are obtained for both particle image shapes.

\subsection{Influence of nonzero particle image displacements on the cross-correlation result}

In the preceding sections, the influence of the particle image size and shape on the cross-correlation result has been investigated only for zero-displaced particle images. The influence of nonzero particle image displacements on the crosscorrelation result is discussed in the following.

As a basis, 500 synthetic double-frame images, each consisting of 5 Gaussian, ring- or plateau-shaped particle images, are generated without image noise (see also Sect. 3). Particle images assume a constant diameter of $D_{P I}=60 \mathrm{px}$. Between both frames, particle images are displaced uniformly by several non-fractional values that range from $\Delta d=1 \mathrm{px}$ to $\Delta d=60 \mathrm{px}=D_{P I} \approx 0.25 \cdot l$ in horizontal 
direction. The cross-correlation evaluation is performed in the commercial software DaVis 8.4 (LaVision $\mathrm{GmbH}$ ) and is analysed with regard to the displacement estimation error, the detectability and the deviation between the calculated and the specified particle image displacement.

Figure 17 shows the displacement estimation error as a function of the magnitude of the displacement vector $\Delta d$, i.e. the horizontal displacement, for Gaussian, ring- and plateaushaped particle images.

Compared to zero-displaced particle images (non-filled markers; see also Fig. 8 in Sect. 4.1), the displacement estimation error slightly increases for particle image displacements of $\Delta d \geq 1 \mathrm{px}$ for all considered particle image shapes. Figure 17 also shows that the displacement estimation error slightly increases with increasing particle image displacement for Gaussian and ring-shaped particle images. Qualitatively, this is in agreement with the development of the estimation error for Gaussian particle images of $D_{P I}=2 \mathrm{px}$ (Westerweel 1997). This increase can be associated with an increase in the second term of the displacement estimation error $\epsilon_{2}$ [see also (6)], as shown in Fig. 18. Here, the deviations of $\epsilon_{1}$ and $\epsilon_{2}$ for nonzero displacements relative to zero displacements are plotted as a function of the magnitude of the particle image displacement.

Figure 18 shows that the first estimation error term $\epsilon_{1}$ is insensitive to a nonzero displacement for all particle image shapes considered. In contrast to this, the second term $\epsilon_{2}$ increases with increasing particle image displacement for Gaussian and ring-shaped particle images. For plateaushaped particle images, the second term $\epsilon_{2}$ is generally larger for nonzero particle image displacements than for a zero displacement, but remains constant over the whole considered particle image displacement range. This is a result of the plateau shape of the particle image that leads to a broadening of the displacement correlation peaks in comparison with those of Gaussian and ring-shaped particle images (see also

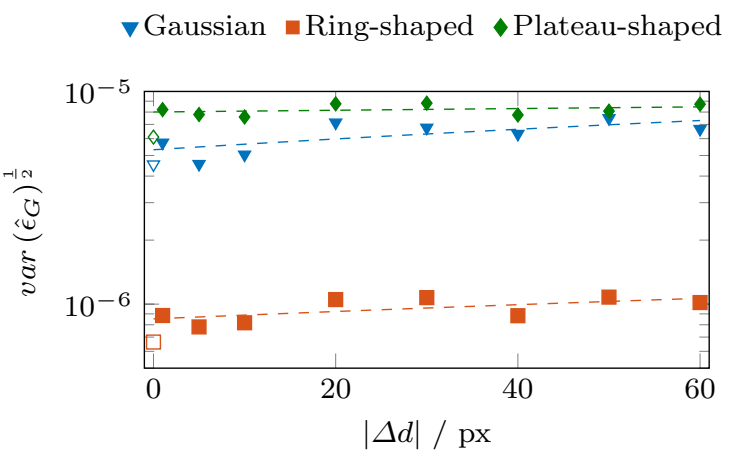

Fig. 17 Estimation error as a function of the particle image displacement for Gaussian, ring- and plateau-shaped particle images of $D_{P I}=60 \mathrm{px}$. Non-filled markers indicate the error estimate for $\Delta d=0$. Dashed lines denote fits to the respective data

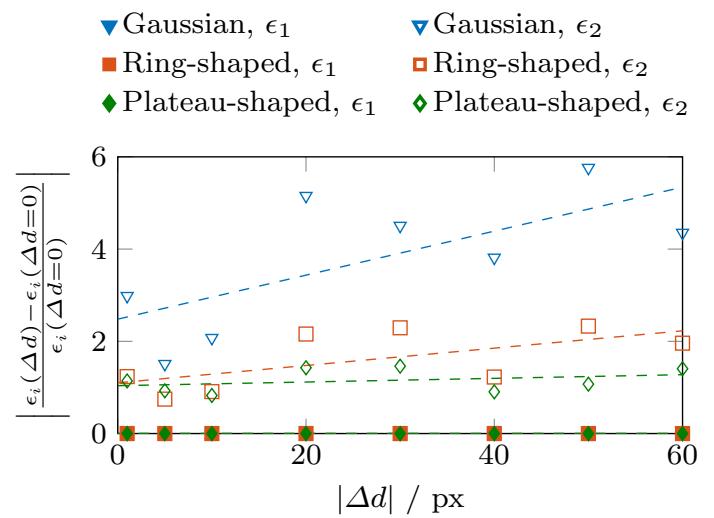

Fig. 18 Estimation error components $\epsilon_{1}$ (filled) and $\epsilon_{2}$ (non-filled) as a function of the particle image displacement for Gaussian, ring- and plateau-shaped particle images of $D_{P I}=60 \mathrm{px}$. Dashed lines denote fits to the respective data

Fig. 7). Obviously, this makes the correlation result insensitive against errors that result from a nonzero particle image displacement. Nevertheless, it should be noted that the highest displacement estimation errors are generally observed for plateau-shaped particle images . Overall, for ring-shaped particle images, the estimation error is approximately one order of magnitude smaller than for Gaussian and plateaushaped particle images over the whole range of considered particle image displacements.

Figure 19 shows the detectability $D$ as a function of the particle image displacement for Gaussian, ring- and plateaushaped particle images.

Compared to zero-displaced particle images of the same size (non-filled markers; see also Fig. 11 in Sect. 4.2), the respective detectability is slightly lower for each particle image shape. A comparison of the detectability evolution for different displacement values reveals that the detectability

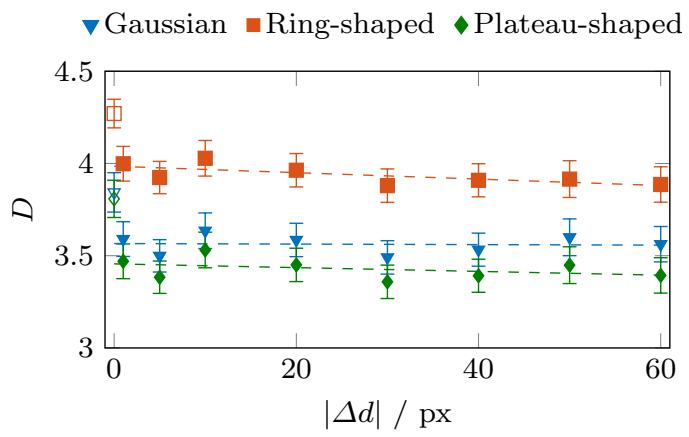

Fig. 19 Detectability $D$ with $95 \%$ confidence interval as a function of the particle image displacement for Gaussian, ring- and plateaushaped particle images of $D_{P I}=60 \mathrm{px}$. Non-filled markers indicate the detectability for $\Delta d=0$. Dashed lines denote fits to the respective data 
is insensitive against a particle image displacement of up to one particle image diameter for all considered particle image shapes. Overall, the detectability for ring-shaped particle images is slightly larger than for Gaussian and plateau-shaped particle images for all considered displacement values.

Figure 20 shows the deviations of the calculated displacements $\delta d$ to the specified displacements $\Delta d$ in horizontal direction as a function of the specified displacement magnitude. For this, the vector results of all 500 double-frame images are averaged for each particle image shape and displacement value, respectively.

Interestingly, calculated displacements of ring-shaped particle images seem to show negligible deviations. In contrast to this, calculated displacements of Gaussian and plateau-shaped particle images deviate from the specified displacements. For Gaussian particle images, the standard deviations are significantly larger compared to plateaushaped particle images for displacements of $\Delta d \geq 5 \mathrm{px}$. Nonzero displacements of Gaussian particle images lead to an increase in $\epsilon_{2}$, which is less significant for plateau-shaped particle images (see also Fig. 18), resulting in larger standard deviations of the calculated particle image displacements.

\section{Experimental application}

The previous study shows that the usage of particles with an image size larger than $10 \mathrm{px}$ leads to a reduced displacement estimation error, if they assume a ring-shaped particle image instead of a plateau or Gaussian shape. Such particle images are encountered in a refractive index matched suspension with surface labelled suspension particles. We demonstrate in the following that such suspension systems allow to study in detail the bulk behaviour of suspensions of up to 5\% volume fraction by means of $\mu$ PIV. For this, PMMA particles of

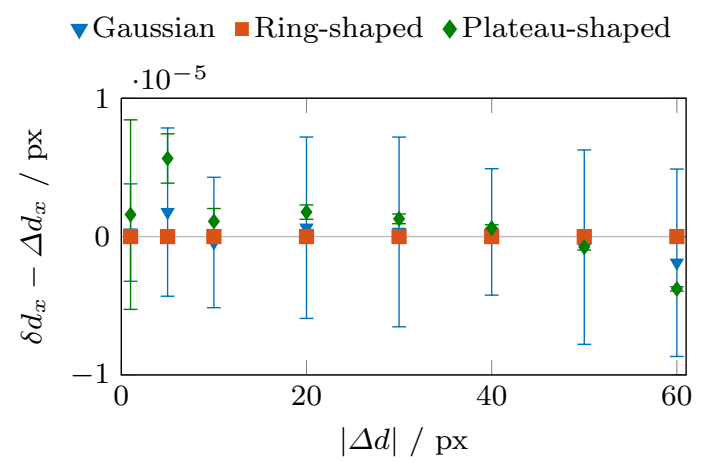

Fig. 20 Deviation between calculated and specified particle image displacement in horizontal direction as a function of the particle image displacement magnitude for Gaussian, ring- and plateaushaped particle images of $D_{P I}=60 \mathrm{px}$
$D_{P I}=60 \mu \mathrm{m}$ diameter that assume ring-shaped images are suspended in a ternary carrier liquid. Flow velocities of both phases of the suspension flow are determined. The results are discussed in Sect. 5.4. These show that a spatial resolution beyond the particle image size can be reached through ensemble averaging to reveal the suspension bulk dynamics. Through refractive index matching and a labelling of only the suspension particle surfaces, an enhanced optical accessibility of the suspension flow could be reached. It should be noted that the intensity signal of ring-shaped particle images is reduced compared to fully labelled particles of the same size. Thus, a suitable illumination source is needed to obtain a sufficient intensity signal. However, in the present study a similar signal-to-noise ratio is obtained for ring-shaped particle images and for plateau-shaped particle images of the same size as well as for standard PIV tracer particles. In this way, it was possible to measure velocity profiles of the suspension carrier liquid simultaneously with those of suspension particles. Measurement results of the one-phase flow with refractive index matched carrier liquid are compared to measurements of the liquid and particle phase of the suspension flow. For this, flow field results for both phases are derived from a single recording, i.e. at the same time.

\subsection{Experimental set-up}

A straight microchannel with trapezoidal cross section, a channel height of $h=580 \mu \mathrm{m}$ and a top and bottom width of $578 \mu \mathrm{m}$ and $321 \mu \mathrm{m}$, respectively, is used (Micronit $\mathrm{GmbH}$ ). The inclination of the microchannel sidewalls results from the fabrication process. A schematic of the microchannel is given in Fig. 21 to illustrate the measurement position.

The suspension consists of a ternary carrier liquid of distilled water, glycerine and ammoniumthiocyanate (Bailey and Yoda 2003) and $60 \mu \mathrm{m}$ PMMA particles that are labelled as already described in Sect. 3. The carrier liquid has a density of $\rho_{l}=1178.07 \pm 0.37 \mathrm{~kg} \mathrm{~m}^{-3}$, a refractive index of $n_{D, l}=1.489$ and a dynamic viscosity of $0.00596 \pm 0.00011$ Pas. PMMA particles (Microbeads Spheromers CA60) assume a density of $\rho_{p}=1200 \mathrm{~kg} \mathrm{~m}^{-3}$ and a refractive index of $n_{D, p}=1.4895 \pm 0.0035$. Thus, the deviations between carrier liquid and particle properties are less than $2 \%$ in density and $0.3 \%$ in refractive index. A nominal volumetric concentration of $\phi=5 \%$ is used throughout the experiments. Standard polystyrene tracer particles of $1.19 \pm 0.03 \mu \mathrm{m}$ diameter (microParticles $\mathrm{GmbH}$ ) are added to the suspension. The same tracer particles are also used for reference measurements of the one-phase flow. For both reference and suspension flow measurements, the same batch of carrier liquid is used.

The experimental set-up consists of an epifluorescent microscope (Nikon Eclipse LV100) that is used in combination with an infinity-corrected objective lens (20X 


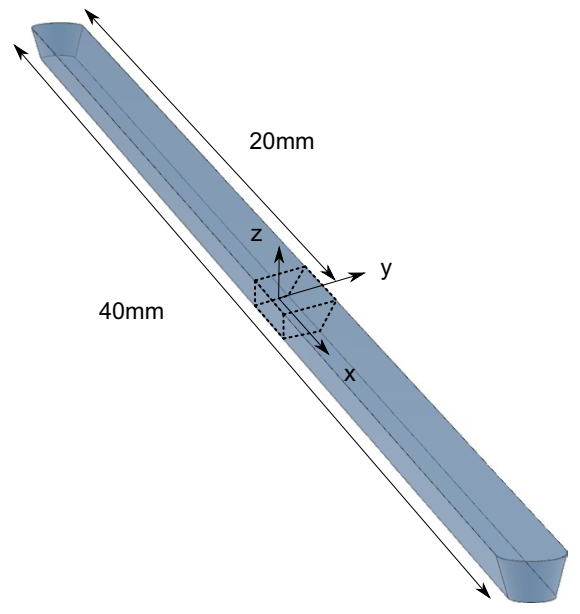

Fig. 21 Schematic figure of the microchannel device including the region of interest shown with black dashed lines

Nikon CFI60 TU Plan Epi ELWD) of $M=20$ magnification and a numerical aperture of $\mathrm{NA}=0.4$. For flow field measurements, a double-pulsed Nd:YAG laser of type Litron Nano S 65-15 PIV and a double-frame CCD camera of type LaVision Imager pro SX is used. The camera has a resolution of $2058 \times 2456 \mathrm{px}$, which results in a field of view of $0.57 \mathrm{~mm} \times 0.48 \mathrm{~mm}$. For image acquisition and evaluation the commercial software DaVis 8.4 (LaVision $\mathrm{GmbH}$ ) is used.

\subsection{Experimental procedure}

All measurements are taken at a bulk Reynolds number of $\operatorname{Re}_{b}=\left(u_{b} \cdot h\right) / v=0.71$. Here, $u_{b}$ denotes the bulk fluid velocity calculated from the volume flow rate and the measured microchannel cross section and $v$ denotes the kinematic viscosity of the ternary carrier liquid, based on the measured dynamic viscosity and density (see Sect. 5.1). Resulting velocity fields of the suspension flow are compared to velocity results of the one-phase flow at the same bulk Reynolds number. As the depth of correlation (Olsen and Adrian 2000) assumes $\delta_{D o C} \approx 16 \mu \mathrm{m}$ for $1.19 \mu \mathrm{m}$ PIV tracer particles in combination with the prescribed optical configuration, measurements are taken at 25 different z-planes with a spatial distance of $d z \approx 22 \mu \mathrm{m}$ from each other to prevent an overlapping of measurement volumes. At every measurement plane, 500 double-frame images are recorded with a frequency of $4 \mathrm{~Hz}$. An interframing time of $d t=750 \mu \mathrm{s}$ is used.

\subsection{Image pre-processing and evaluation}

Image pre-processing steps are performed before the actual cross-correlation evaluation to increase the signal-to-noise ratio of the particle images. The image pre-processing procedure that is applied in the present study is described in Sect. 5.3.1.

In the experimental study, tracer particles with small particle image diameters and suspension particles with large particle image diameters are suspended to the flow to measure bulk slip velocities. However, for the crosscorrelation evaluation both types of particle images have to be segmented from each other before processing. The procedure that is used to do this is described in Sect. 5.3.2.

Finally, pre-processed and segmented particle images are evaluated by cross-correlation. The parameters that are used for this are described in Sect. 5.3.3 for both phases individually.

\subsubsection{General image pre-processing}

The general image pre-processing procedure consists of three steps. In a first step, a minimum intensity image of the first 19 images of each recording sequence is generated and subtracted from each recorded image of the same recording sequence. This reduces stationary background noise and reflections. In a second step, a spatial sliding average filter of 3 px edge length including a Gaussian weighting function is applied to each image to reduce saltand-pepper noise. In a third step, a spatial subtract sliding average filter of 200 px edge length is applied to each image to further enhance the signal-to-noise ratio. This results in an increased intensity gradient at the ring-shaped suspension particle image rim and, therefore, facilitates the detection of ring-shaped suspension particle images. The segmentation procedure is described in the following section.

\subsubsection{Particle image segmentation}

Pre-processed images contain signals from both PIV tracers and ring-shaped suspension particle images. To segment individual particle groups, an in-house MATLAB code is used. The segmentation procedure is based on Anders et al. (2019).

The first step during image segmentation is to detect large suspension particle images, specifically their centre points and radii. This is done for both frames of a double-frame image. The centre point and radius information are used to define one masking function that is applied on both frames. With this, intensity information of the area that is covered 
by suspension particle images within the original, pre-processed images are extracted.

The extraction of suspension particle images leaves spaces with zero intensity in the original images (that now contain only signals from PIV tracer particles). As shown by Anders et al. (2019), this can lead to a velocity bias, when using cross-correlation-based evaluation methods. To overcome this, a spectral random masking algorithm is proposed. This utilizes the image noise information of the vicinity of suspension particle images and combines these with a bilinear fit of the spatial intensity information to fill the regions, where suspension particle images are extracted.

Intensity information of extracted suspension particle images are stored in a separate double-frame image. As these images may contain also intensity information resulting from small PIV tracer particles, further pre-processing steps are performed to obtain solely the intensity signals of suspension particles. Specifically, a sliding average filter with 7 px edge length and a Gaussian weighting function is utilized first. Afterwards, a bandwidth filter is applied that retains only structures with a length between 7 px and 25 px.

\subsubsection{Cross-correlation evaluation}

Based on the pre-processed and segmented particle image recordings, velocity fields are obtained by performing sumof-correlation cross-correlations with the commercial DaVis software (LaVision $\mathrm{GmbH}$ ). A multi-pass method (Willert 1996) with decreasing interrogation window sizes of $256 \times 256$ px during the first pass and $128 \times 128$ px during both the second and third pass are utilized for the evaluation of the carrier liquid flow, i.e. for PIV tracer particle images. As suspension particles assume a particle image diameter of $D_{P I} \approx 270 \mathrm{px}$, interrogation window sizes are set to $512 \times 512 \mathrm{px}$ during the first pass and $256 \times 256 \mathrm{px}$ during the second and third pass. This is suitable, as we showed in Sect. 4.3 that the estimation error of ring-shaped particle images is insensitive to intersection at the interrogation window borders. For all cross-correlation evaluations, a $50 \%$ overlap of interrogation windows is used. Vector postprocessing is performed to eliminate erroneous velocity data. Specifically, vector results with a detectability below 2 are deleted and, additionally, an universal outlier detection is applied with a $5 \times 5$ median filter. This median filter defines velocity vectors as outliers if the value of a velocity component exceeds the median of the surrounding velocity data by an absolute value that corresponds to one times the median absolute deviation. Emerging empty vector spaces are filled with velocity information resulting from the 2 nd, 3 rd or 4th highest correlation peak, if they fulfil the median outlier criterion instead. In the end, all remaining empty vector spaces are filled up by interpolation. Overall, $4-6 \%$ of all vector information are replaced in an evaluation. 90\% of all replaced vectors are interpolated.

\subsection{Experimental results}

This section discusses experimental flow field results that are obtained by $\mu$ PIV measurements on a ternary carrier liquid and particles of a suspension flow (Sect. 5.4.1). An error analysis of the $\mu \mathrm{PIV}$ measurement results is performed in Sect. 5.4.2.

\subsubsection{Flow field results}

The performed flow field measurements demonstrate the ability to use ring-shaped particle images in combination with standard PIV tracer particles to determine simultaneously velocity fields from both phases of a suspension flow by means of $\mu$ PIV.

PIV vector fields are derived through ensemble averaging of 500 double-frame images. Measurements are taken at 25 equidistantly spaced measurement planes. Velocity results of the one-phase flow (OPF) are compared against the suspension carrier liquid (SCL) and the suspension particles (SP) velocities. Velocity fields are averaged in streamwise direction. For the one-phase flow, this results in the streamwise averaged velocity field that is shown over the cross section in Fig. 22. The microchannel walls are sketched as crosshatched regions, indicating the trapezoidal cross-sectional shape.

The velocity profile assumes its maximum at $z \approx 335 \mu \mathrm{m}$ (horizontal dashed line in Fig. 22). The corresponding velocity profile is shown in Fig. 23a for the one-phase flow (OPF), the suspension carrier liquid (SCL) and the suspension particle (SP) flow.

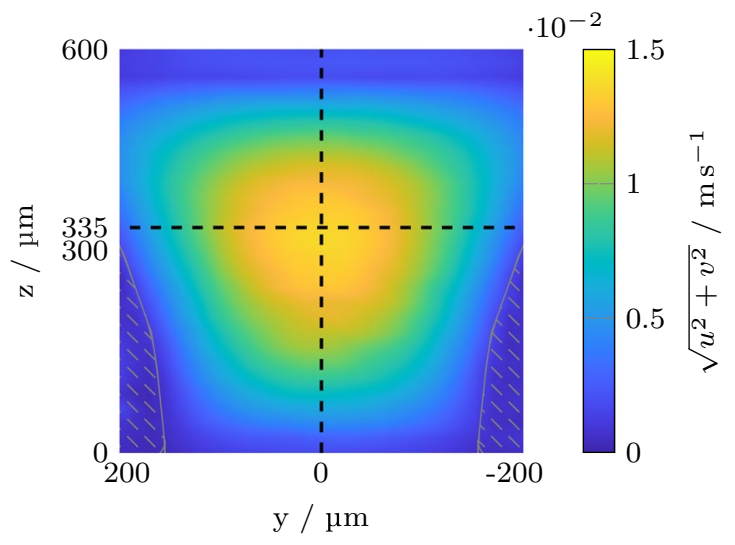

Fig. 22 Streamwise averaged velocity magnitudes in the microchannel cross-section obtained from $\mu$ PIV measurements of the one-phase flow (OPF). Velocity profiles are analysed on xy- and xz-planes as indicated here by dashed lines. Channel walls are sketched as crossatched regions 


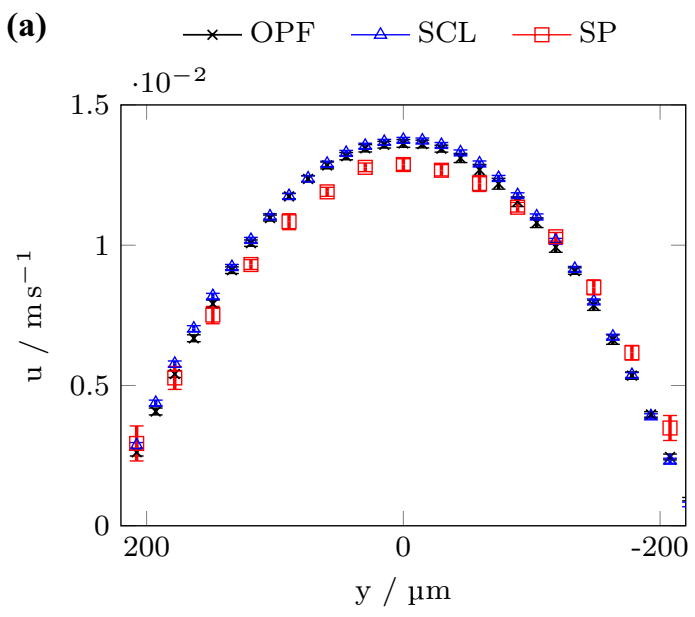

(b)

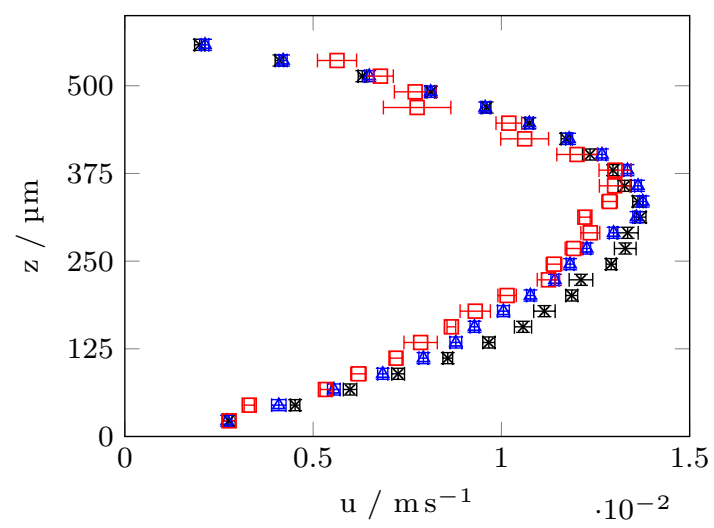

Fig. 23 Velocity profiles obtained from $\mu \mathrm{PIV}$ measurements of the one-phase flow (OPF), the suspension carrier liquid (SCL) and the suspension particle (SP) flow a on the xy-plane at $z=335 \mu \mathrm{m}$ above the channel bottom and $\mathbf{b}$ on the xz-plane in the channel bisector at $y=0 \mu \mathrm{m}$. Error bars indicate the uncertainty resulting from streamwise averaging and the particle image displacement detection uncertainty at the upper limit of the $68.5 \%$ confidence interval

Figure $23 \mathrm{a}, \mathrm{b}$ shows the velocity profiles of the onephase flow, the suspension carrier liquid and the suspension particle flow, respectively, at $z \approx 335 \mu \mathrm{m}$ and at the microchannel bisector, i.e. $y=0 \mu \mathrm{m}$. Error bars indicate the overall measurement uncertainty that results from streamwise averaging and the uncertainty estimation of the particle image displacement detection at the upper limit of the $68.5 \%$ confidence interval. Further details on the error analysis are given in Sect. 5.4.2. The in-plane velocity profile of the one-phase flow (OPF) as shown in Fig. 23a assumes a parabolic shape with a maximum velocity of $u=0.0136 \pm 0.0001 \mathrm{~m} \mathrm{~s}^{-1}$. At the microchannel side walls, velocities deviate from zero, which results from the presence of near-wall tracer particles that move with a nonzero velocity. Due to spatial averaging inside near-wall interrogation windows and a high-velocity gradient in these

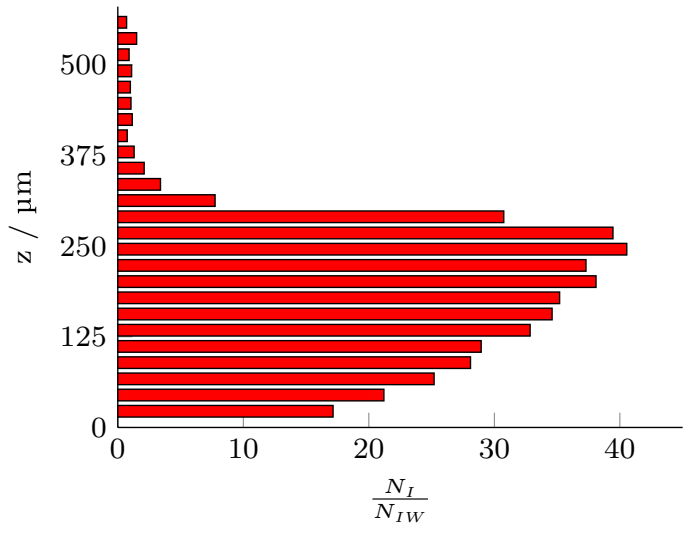

Fig. 24 Average number of segmented suspension particle images $N_{I}$ divided by the number of interrogation windows in streamwise direction $N_{I W}$ along the microchannel height at the microchannel bisector

regions, the presence of those tracer particles results in velocities that assume nonzero values.

A comparison of the in-plane velocity profiles at $z=335 \mu \mathrm{m}$ between the one-phase flow (OPF) and the suspension carrier liquid (SCL) flow shows no significant differences. Suspension particles (SP) generally tend to lag behind the suspension carrier liquid (SCL) up to $7.4 \%$. An error analysis shows that this can be confirmed within a $68.5 \%$ confidence interval (see also Sect. 5.4.2). Liquid-particle slip velocities are expected, due to a suspension particle size which assumes a similar order of magnitude as the microchannel height $\left(D_{P} / h=0.1\right)$ and is a well-known effect in Poiseuille flow for neutrally buoyant particles (Brenner 1966; Feng et al. 1994; Guazzelli and Morris 2011). Numerical investigations of Loisel et al. (2015) showed similar trends of such relative velocities compared to our results in suspension flows with homogeneously distributed particles and volume concentrations of up to 5\%. Their results indicate that the particle slip velocity is rather a function of the Reynolds number than of the particle volume concentration in the investigated particle volume concentration regime.

The velocity profiles in the xz-plane on the microchannel bisector $(y=0 \mu \mathrm{m}$, see also vertical dashed line in Fig. 22), are shown in Fig. 23b. In the lower part of the microchannel for $z<300 \mu \mathrm{m}$, the suspension carrier liquid (SCL) lags behind the one-phase flow (OPF) up to $12 \%$. In contrast to this, similar flow velocities can be observed in the top region of the microchannel for $z \gtrsim 300 \mu \mathrm{m}$. Suspension particles (SP) in turn assume a generally lower velocity than the suspension carrier liquid (SCL) at the microchannel centre, as already discussed above. This can be qualitatively understood when looking at the particle image density over the channel height, being an indication for the particle concentration distribution, see Fig. 24. The number of segmented suspension particle images $N_{I}$ within the field of 
view during the whole time series is divided by the amount of interrogation windows $N_{I W}$ of the resulting vector field in streamwise direction. These values are plotted for the microchannel bisector as a function of the microchannel height. A particle image is assigned to an interrogation window, if its centre point is located inside the corresponding interrogation window borders. Therefore, the ratio $N_{I} / N_{I W}$ indicates how many particle images contribute on average to the displacement correlation peak of a single interrogation window. Clearly, particles are distributed inhomogeneously over the microchannel height. We anticipate that a particle drift away from the channel top is induced at the inlet region where a sharp corner flow is induced, accelerating particles towards the channel bottom. It may be noted that the data of Fig. 24 display the particle image number density within each measurement volume.

For $z \gtrsim 300 \mu \mathrm{m}$, the amount of particle images per interrogation window assumes on average $N_{I} / N_{I W}<5$ which is below the effective number of particle images which ensures a high detectability in PIV measurements (Keane and Adrian 1992). This becomes evident through increasing standard deviations of suspension particle velocity data for $z>300 \mu \mathrm{m}$ in Fig. 23b. As the particle concentration is significantly reduced in this region, the velocity of the suspension carrier liquid assumes that of the one-phase flow here.

\subsubsection{Error analysis of the flow field results}

Different uncertainty quantification methods are available and comprehensive overviews of these approaches can be found in Sciacchitano et al. (2015) and Sciacchitano (2019). Generally, different methods can be categorized into statistical and, following Sciacchitano (2019), direct and indirect methods.

Statistical approaches calculate the deviation of a flow quantity from its time or spatial average to determine the measurement uncertainty (Sciacchitano and Wieneke 2016). Therefore, the statistical calculations are applied on the velocity or displacement results.

Direct uncertainty quantification approaches are the particle disparity (Sciacchitano et al. 2013) or the correlation statistics (Wieneke 2015) approach. Both methods use the displacement field of a PIV evaluation to remove the image shift between two corresponding frames. As measurement errors result in mismatches between such frames, either the contribution of individual particle images or individual pixels inside an interrogation window to the displacement correlation peak, are used to calculate the measurement uncertainty. Other examples for direct methods are the moment of correlation plane (Bhattacharya et al. 2018) and the error sampling approach (Smith and Oberkampf 2014). The moment of correlation plane approach calculates the standard uncertainty from the second moment of the generalized cross-correlation that is convolved with a two-dimensional Gaussian fit function. The error sampling approach suggests to repeat an experiment several times while systematically varying different aspects of the experiment that affect the measurement error to receive an accurate estimate of the overall error.

Indirect methods are the uncertainty surface approach (Timmins et al. 2012) and the correlation signal-to-noise ratio metrics (Charonko and Vlachos 2013; Xue et al. 2014) approach. The uncertainty surface approach utilizes synthetic data that are representative for the corresponding measurement situation to determine an uncertainty surface, from which the resulting measurement uncertainty is calculated. In contrast to this, the correlation SNR metrics approach uses an empirical equation to calculate the measurement uncertainty at the upper and lower limit of the $68.5 \%$ and $95 \%$ confidence intervals from differently defined signal-to-noise ratios of the correlation plane. With this, Xue et al. (2014) showed that the error magnitude of PIV data is not necessarily normally distributed.

It shall be noted that not all of the uncertainty estimation approaches that are described in the preceding are applicable to the experimental results of the present study. One reason for this is the usage of an ensemble-averaged crosscorrelation method that determines particle image displacements from an average that is performed in the correlation plane. Another reason is the usage of large ring-shaped particle images that can lead to displacement correlation peaks where only the correlation peak region can be approximated by a Gaussian function.

In the present study, an error analysis is performed by utilizing two different approaches. Firstly, the spatial distributions of the root mean square errors (RMSE) of the streamwise velocity component are calculated. The second approach determines the measurement uncertainty $U$ of the displacement magnitude at the upper limit (UL) and lower limit (LL) of the $68.5 \%$ and $95 \%$ confidence intervals. These are based on the empirical equation developed by Xue et al. (2014). For this, the correlation map entropy is used as representative correlation SNR metric. Thus, these uncertainty estimates are measures for the random errors of the crosscorrelation evaluations.

For the calculation of the RMSE distributions of the streamwise velocity component, RMS errors of consecutive vector results in streamwise direction are calculated. The resulting RMSE distributions for the one-phase flow (OPF), the suspension carrier liquid (SCL) and the suspension particle (SP) flow, respectively, are shown in Fig. 25a-c as contour plots.

A comparison of Fig. 25a and $b$ reveals that the RMSE of the one-phase and the suspension carrier liquid flow are similar. It may be noted that $25 \mathrm{a}$ and $\mathrm{b}$ are both derived from PIV recordings of $1.19 \mu \mathrm{m}$ fluid tracers. A comparable 

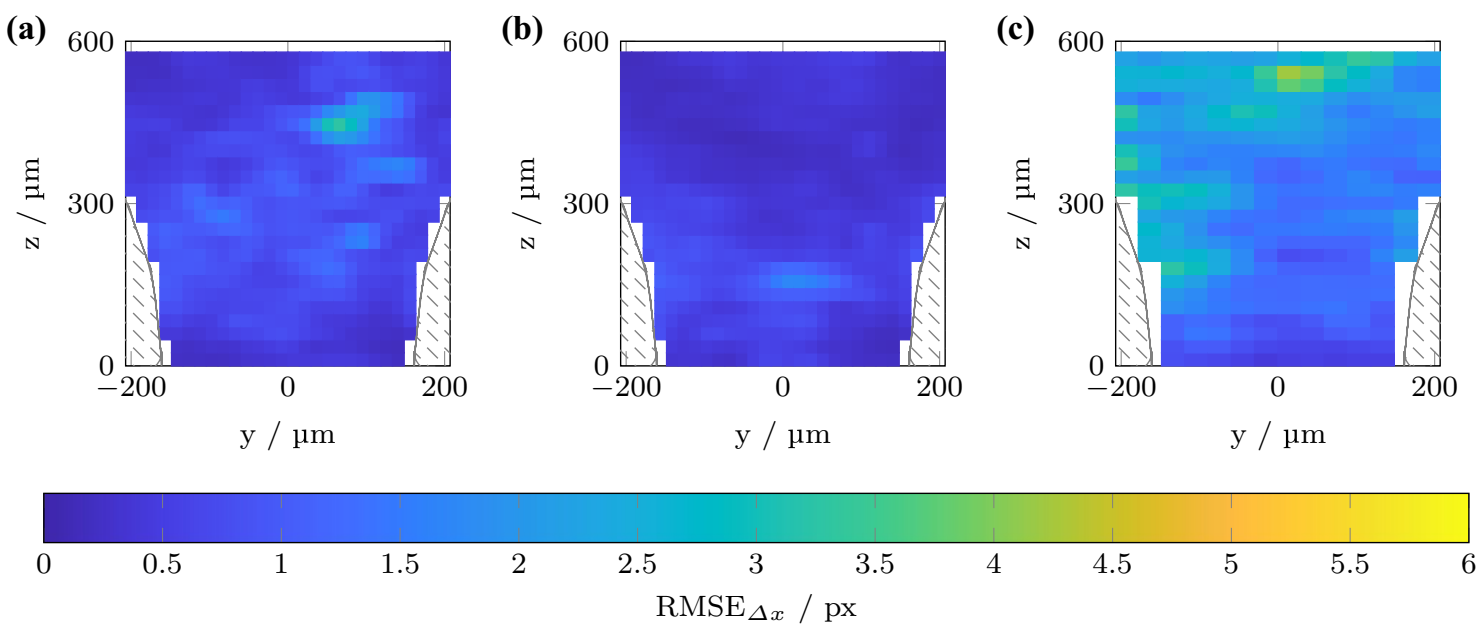

Fig. 25 Root mean square error distributions over the microchannel cross-section for a the one-phase flow (OPF), b the suspension carrier liquid (SCL) flow and $\mathbf{c}$ the suspension particle (SP) flow

RMSE for these flows shows that the separation of fluid tracer and suspension particle images, as described in Sect. 5.3.2, has only a minor influence on the image result. From Fig. 25a-c, average RMSE values are calculated. These are shown in Table 1 in displacement (px) and velocity $\left(\mathrm{mm} \mathrm{s}^{-1}\right)$ dimensions.

The average RMSE of the suspension particle flow is approximately one order of magnitude larger than for the one-phase flow and the suspension carrier liquid flow. This is assumed to result from an inhomogeneous particle distribution over the microchannel cross section (see also Fig. 24) as well as interactions between suspension particles and microchannel walls and particle-particle interactions, which are known to occur in suspension flows with volume concentrations of $5 \%$.

The measurement uncertainties at the upper and lower limit of the $68.5 \%$ and $95 \%$ confidence intervals are calculated for the ensemble-averaged cross-correlation results utilizing the empirical relation developed by Xue et al. (2014). In the present study, the correlation entropy is utilized to calculate uncertainty estimates for each interrogation window. Exemplary distributions of the measurement uncertainties at the upper limit of the $95 \%$ confidence interval are shown in Fig. 26a-c for the one-phase flow (OPF), the suspension carrier liquid (SCL) and the suspension particle (SP) flow at $z=335 \mu \mathrm{m}$.

The resulting uncertainty estimates at the upper and lower limit of the $68.5 \%$ and $95 \%$ confidence intervals are averaged over all interrogation windows of all measurement planes. The results are also shown in Table 1 in displacement $(\mathrm{px})$ and velocity $\left(\mathrm{mm} \mathrm{s}^{-1}\right)$ dimensions. The average uncertainty estimates are at least one order of magnitude smaller than the maximum streamwise velocities. Furthermore, the uncertainty estimates are similar for all particle image shapes considered.

Thus, the presence of a slip velocity between carrier liquid and suspension particles can be confirmed within a $68.5 \%$ confidence interval for which the uncertainty is approximately one order of magnitude smaller than for measured slip velocities (see also the velocity profiles shown
Table 1 Average root mean square error (RMSE) and measurement uncertainty $U$ at the upper limit (UL) and lower limit (LL) of the $68.5 \%$ and $95 \%$ confidence intervals for the one-phase flow (OPF), suspension carrier liquid (SCL) and suspension particle (SP) flow

\begin{tabular}{llll}
\hline & OPF & SCL & SP \\
\hline $\mathrm{RMSE}_{\Delta x} / \mathrm{px}$ & $0.58 \pm 0.21$ & $0.38 \pm 0.13$ & $1.7 \pm 0.5$ \\
$\mathrm{RMSE}_{\Delta u} / \mathrm{mm} \mathrm{s}^{-1}$ & $0.18 \pm 0.065$ & $0.12 \pm 0.040$ & $0.53 \pm 0.017$ \\
$U_{\Delta x, \mathrm{UL}, 68.5} / \mathrm{px}$ & $0.27 \pm 0.00044$ & $0.27 \pm 0.00042$ & $0.27 \pm 0.00092$ \\
$U_{\Delta x, \mathrm{LL}, 68.5} / \mathrm{px}$ & $0.053 \pm 0.000079$ & $0.053 \pm 0.000077$ & $0.053 \pm 0.00019$ \\
$U_{\Delta u, \mathrm{UL}, 68.5} / \mathrm{mm} \mathrm{s}^{-1}$ & $0.084 \pm 0.00013$ & $0.084 \pm 0.00013$ & $0.084 \pm 0.00028$ \\
$U_{\Delta u, \mathrm{LL}, 68.5} / \mathrm{mm} \mathrm{s}^{-1}$ & $0.016 \pm 0.000025$ & $0.016 \pm 0.000024$ & $0.016 \pm 0.000058$ \\
$U_{\Delta x, \mathrm{UL}, 95} / \mathrm{px}$ & $1.75 \pm 0.0028$ & $1.8 \pm 0.0027$ & $1.75 \pm 0.0055$ \\
$U_{\Delta x, \mathrm{LL}, 95} / \mathrm{px}^{-1}$ & $0.020 \pm 0.000033$ & $0.020 \pm 0.000032$ & $0.020 \pm 0.000075$ \\
$U_{\Delta u, \mathrm{UL}, 95} / \mathrm{mm} \mathrm{s}^{-1}$ & $0.54 \pm 0.00087$ & $0.54 \pm 0.00085$ & $0.54 \pm 0.0017$ \\
$U_{\Delta u, \mathrm{LL}, 95} / \mathrm{mm} \mathrm{s}^{-1}$ & $0.0063 \pm 0.000010$ & $0.0063 \pm 0.0000098$ & $0.0063 \pm 0.000023$ \\
\hline
\end{tabular}



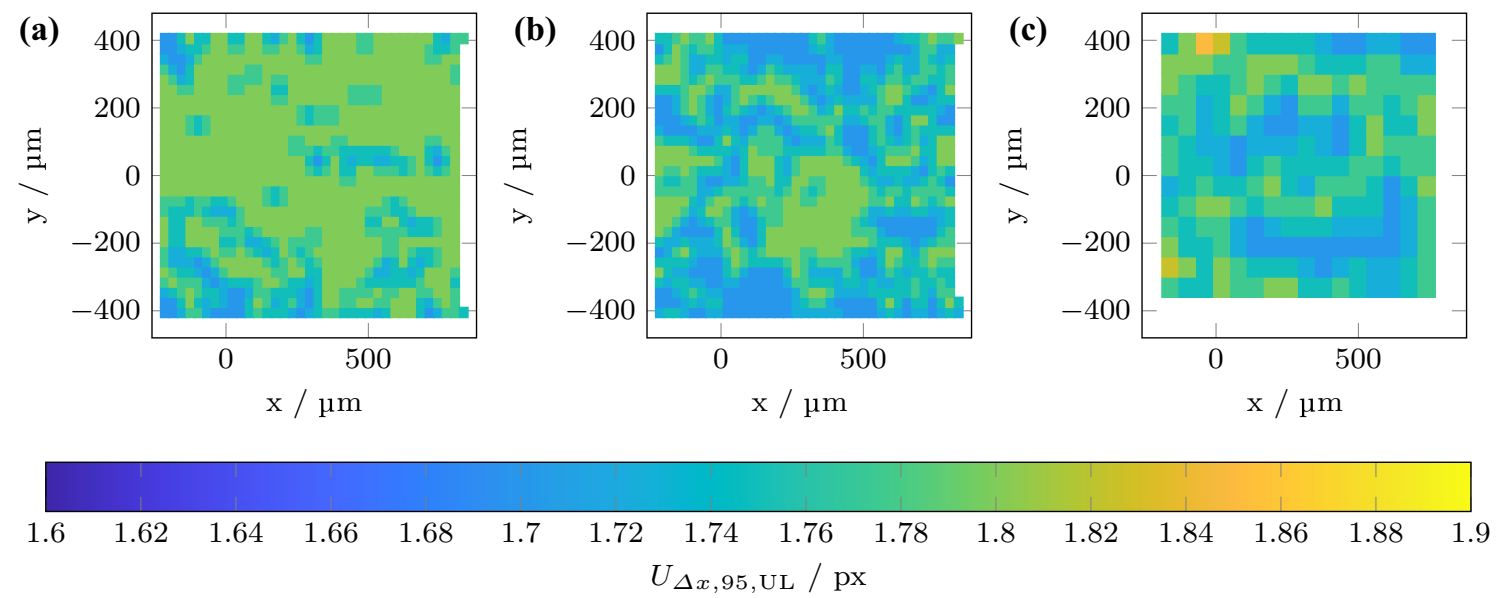

Fig. 26 Measurement uncertainty at the upper limit of the 95\% confidence interval based on the correlation entropy (Xue et al. 2014) for a the one-phase flow, $\mathbf{b}$ the suspension carrier liquid (SCL) flow and $\mathbf{c}$ the suspension particle (SP) flow at $z=335 \mu \mathrm{m}$

in Fig. 23a, b). Furthermore, the usage of large ring-shaped particle images for the cross-correlation evaluation does not lead to a significant increase in the measurement uncertainty within the considered uncertainty bounds.

\section{Conclusion}

Overall, we demonstrate that refractive index matched and surface labelled suspension particles open up the possibility to investigate both suspension and carrier bulk dynamics simultaneously by means of $\mu \mathrm{IV}$. The size sensitivity of Gaussian, ring- and plateau-shaped particle images on crosscorrelation-based PIV evaluations is compared. When particle images become large compared to standard PIV tracer particle images, accuracy and reliability of cross-correlations on resulting standard Gaussian or plateau-shaped particle images are known to decrease. At higher particle volume fractions, the optical accessibility of such suspension flows is usually limited, as well. To overcome this, we perform a refractive index matching of a carrier fluid to PMMA suspension particles. Furthermore, a surface labelling of such suspension particles leads to ring-shaped particle images. The suitability of such particle images with regard to a PIV cross-correlation evaluation is investigated in this work. For all parameter studies, cross-correlation results of synthetic particle images are evaluated by means of displacement estimation error and detectability for particle image diameters between $1 \mathrm{px} \leq D_{P I} \leq 60 \mathrm{px}$. The study is based on Monte Carlo simulations with 500 double-frame images for each particle image diameter. Particle images are cross-correlated using a commercial PIV evaluation algorithm.

It is shown that ring-shaped particle images assume a reduced displacement estimation error for particle image diameters beyond $D_{P I}=10 \mathrm{px}$ compared to Gaussian and plateau-shaped particle images while there is no significant difference in the detectability for different particle image shapes. This reduction in displacement estimation error of ring-shaped particle images is related to increased intensity gradients at the particle image rim in combination with a nearly transparent centre region. This leads to a faster decorrelation and thus reduces the correlation peak width. When image noise is added, the overall estimation error is increased by several orders of magnitude due to an increase in random errors (here an increase in four orders of magnitude was observed for $8.5 \%$ image noise). Thus, special attention has to be spent on the elimination of image noise during pre-processing of experimental data, since image noise generally leads to an increased displacement estimation error for all investigated particle image shapes at various particle image diameters. However, also in this situation ring-shaped particle images assume a reduced displacement estimation error compared to Gaussian and plateau-shaped particle images.

Furthermore, the influence of particle images being intersected at the interrogation window border is investigated. For this, synthetic images with one, three and five intersected particle images out of five within each interrogation window are evaluated for Gaussian, ring- and plateau-shaped particle images. While the displacement estimation error of ring-shaped particle images appears to be insensitive to particle image intersection at the interrogation window border, a strong reduction in estimation error is observed for Gaussian and plateau-shaped particle images with increasing amount of intersected particle images. This can be explained by intensity jumps of particle images that occur at the intersection line along the interrogation window border. These intensity jumps, which are much more prominent for Gaussian and plateau-shaped particle images, lead to a reduction 
of the correlation peak width and hence a reduced estimation error.

When a nonzero particle image displacement is considered, the displacement estimation error increases with the displacement value for Gaussian and ring-shaped particle images. In contrast to this, the displacement estimation error of plateau-shaped particle images is insensitive against a nonzero particle image displacement within the investigated range. Compared to a zero particle image displacement, the displacement estimation error increases for all considered particle image shapes. However, the displacement estimation error is approximately one order of magnitude smaller for ring-shaped particle images than for Gaussian and plateaushaped particle images for all particle image displacements considered.

The quality of a PIV evaluation can be evaluated from the displacement estimation error and the detectability. The detectability is known to depend on the signal-to-noise ratio, the effective number of particle images, the correlated ensemble size and the particle image density. In the present study, the first three parameters are fixed for all particle image shapes. Therefore, no significant difference in detectability is observed between different particle image shapes for particle images located inside the interrogation window. This holds also true for particle images of $D_{P I}=60 \mathrm{px}$, when a nonzero particle image displacement is considered. However, the detectability is found to decrease with increasing particle image size. This is due to a rise in the particle image density $N_{p p p}$ associated with an increasing particle image diameter. Secondly, a decrease in detectability is observed for particle image diameters below $D_{P I}=10 \mathrm{px}$ with image noise of $8.5 \%$. That is, because intensity values are averaged over a full pixel leading to a reduction in intensity peaks, especially for small particle images. This leads to a significant decrease in signal-to-noise ratio and hence a reduced detectability.

Furthermore, the detectability is investigated for a growing number of intersected particle images, keeping the total number of particle images within the interrogation window constant. Here, a nonlinear behaviour is observed with a minimum in detectability for a similar number of particle images located inside the interrogation window and on the interrogation window border. We assume that for $K_{5}$ being approximately equal to half the total amount of particle images per interrogation window the probability for particle images inside the interrogation window to correlate with intersected particle images is increased, leading to an increase in secondary correlation peak values.

Summed up, the usage of refractive index matched and surface labelled particles not only enhances optical accessibility, but also leads to a reduction of the displacement estimation error and a similar or even better detectability compared to Gaussian or plateau-shaped particle images.
This holds true also for situations where intersected particle images at interrogation window borders occur.

Refractive index matching led to an enhanced optical accessibility of the system. Combined with a labelling, the accuracy and reliability of the $\mu \mathrm{PIV}$ evaluation are enhanced compared to Gaussian particle images. We demonstrate both theoretically and experimentally that such particle systems are suitable to study the bulk dynamics of suspension flows. Measurements are performed with surface labelled and refractive index matched suspension particles that assume ring-shaped particle images. Hence, the particle bulk dynamics of a 5 vol\% suspension is measured together with the carrier liquid flow and the measurement uncertainty is estimated. By this, liquid-particle slip velocities are determined.

Overall, we demonstrate that $\mu \mathrm{IV}$ measurements on ringshaped particle images bring along advantageous properties, from both a theoretical and practical point of view. These are demonstrated to be an important step to perform $\mu$ PIV measurements in a variety of suspension flows. For studies of even higher volume fractions, a well-defined temperature control will be required to achieve a matching of the refractive index between particles and carrier liquid up to the fourth digit (Wiederseiner et al. 2011).

Acknowledgements Financial support from the German research foundation (DFG-SPP 2045) is gratefully acknowledged (Jeanette Hussong HU2264/3-1 I HU2264/3-2 and Harald Kruggel-Emden KR3446/14-1 | KR3446/14-2).

Funding Open Access funding enabled and organized by Projekt DEAL.

Open Access This article is licensed under a Creative Commons Attribution 4.0 International License, which permits use, sharing, adaptation, distribution and reproduction in any medium or format, as long as you give appropriate credit to the original author(s) and the source, provide a link to the Creative Commons licence, and indicate if changes were made. The images or other third party material in this article are included in the article's Creative Commons licence, unless indicated otherwise in a credit line to the material. If material is not included in the article's Creative Commons licence and your intended use is not permitted by statutory regulation or exceeds the permitted use, you will need to obtain permission directly from the copyright holder. To view a copy of this licence, visit http://creativecommons.org/licenses/by/4.0/.

\section{References}

Adrian RJ (1991) Particle-imaging techniques for experimental fluid mechanics. Annu Rev Fluid Mech 23(1):261-304. https://doi.org/ 10.1146/annurev.fl.23.010191.001401

Adrian RJ, Westerweel J (2011) Particle image velocimetry. Aerospace Series. Cambridge University Press, Cambridge

Anders S, Noto D, Seilmayer M, Eckert S (2019) Spectral random masking: a novel dynamic masking technique for PIV in 
multiphase flows. Exp Fluids 60(4):1-6. https://doi.org/10.1007/ s00348-019-2703-8

Bailey BC, Yoda M (2003) An aqueous low-viscosity density- and refractive index-matched suspension system. Exp Fluids 35(1):13. https://doi.org/10.1007/s00348-003-0598-9

Bhattacharya S, Charonko JJ, Vlachos PP (2018) Particle image velocimetry (PIV) uncertainty quantification using moment of correlation (MC) plane. Meas Sci Technol 29(11). https://doi.org/10. 1088/1361-6501/aadfb4

Blanc F, Lemaire E, Meunier A, Peters F (2013) Microstructure in sheared non-Brownian concentrated suspensions. J Rheol 57(1):273-292. https://doi.org/10.1122/1.4766597

Brenner H (1966) Hydrodynamic resistance of particles at small Reynolds numbers. In: Drew TB, Hoopes JW, Vermeulen T (eds) Advances in chemical engineering, advances in chemical engineering, vol 6. Academic Press, pp 287-438. https://doi.org/10. 1016/S0065-2377(08)60277-X

Brockmann P, Hussong J (2021) On the calibration of Astigmatism particle tracking velocimetry for suspensions of different volume fractions. Exp Fluids 62(1):1-11. https://doi.org/10.1007/ s00348-020-03120-4

Brockmann P, Kazerooni HT, Brandt L, Hussong J (2020) Utilizing the ball lens effect for astigmatism particle tracking velocimetry. Exp Fluids 61(2):1-19. https://doi.org/10.1007/s00348-020-2900-5

Byron ML, Variano EA (2013) Refractive-index-matched hydrogel materials for measuring flow-structure interactions. Exp Fluids 54(2). https://doi.org/10.1007/s00348-013-1456-z

Charonko JJ, Vlachos PP (2013) Estimation of uncertainty bounds for individual particle image velocimetry measurements from crosscorrelation peak ratio. Meas Sci Technol 24(6). https://doi.org/10. 1088/0957-0233/24/6/065301

Chen B, Mikami F, Nishikawa N (2005) Experimental studies on transient features of natural convection in particles suspensions. Int J Heat Mass Transf 48(14):2933-2942. https://doi.org/10.1016/j. ijheatmasstransfer.2004.11.016

Coupland JM, Pickering CJ (1988) Particle image velocimetry: estimation of measurement confidence at low seeding densities. Opt Lasers Eng 9(3-4):201-210. https://doi.org/10.1016/S01438166(98)90003-3

Eckstein A, Vlachos PP (2009) Digital particle image velocimetry (DPIV) robust phase correlation. Meas Sci Technol 20(5). https:// doi.org/10.1088/0957-0233/20/5/055401

Eckstein AC, Charonko J, Vlachos P (2008) Phase correlation processing for DPIV measurements. Exp Fluids 45(3):485-500. https:// doi.org/10.1007/s00348-008-0492-6

Feng J, Joseph DD, Hu HH (1994) Direct simulation of initial value problems for the motion of solid bodies in a Newtonian fluid. Part 2. Couette and Poiseuille flows. J Fluid Mech 277:271-301. https://doi.org/10.1017/S0022112094002764

Fitzgibbon S, Spann AP, Qi QM, Shaqfeh ES (2015) In vitro measurement of particle margination in the microchannel flow: effect of varying hematocrit. Biophys J 108(10):2601-2608. https://doi. org/10.1016/j.bpj.2015.04.013

Funatani S, Fujisawa N, Ikeda H (2004) Simultaneous measurement of temperature and velocity using two-colour LIF combined with PIV with a colour CCD camera and its application to the turbulent buoyant plume. Meas Sci Technol 15(5):983-990. https://doi.org/ 10.1088/0957-0233/15/5/030

Guazzelli E, Morris JF (2011) A physical introduction to suspension dynamics, vol 45. Cambridge University Press

Haam SJ, Brodkey RS, Fort I, Klaboch L, Placnik M, Vanecek V (2000) Laser Doppler anemometry measurements in an index of refraction matched column in the presence of dispersed beads: part I. Int J Multiphase Flow 26(9):1401-1418. https://doi.org/ 10.1016/S0301-9322(99)00094-4
Hassan YA, Dominguez-Ontiveros EE (2008) Flow visualization in a pebble bed reactor experiment using PIV and refractive index matching techniques. Nucl Eng Des 238(11):3080-3085. https:// doi.org/10.1016/j.nucengdes.2008.01.027

Keane RD, Adrian RJ (1990) Optimization of particle image velocimeters. I. Double pulsed systems Optimization of particle image velocimeters. Part I: Double pulsed systems. Meas Sci Technol Meas Sci Technol 1(1):1202-1215

Keane RD, Adrian RJ (1992) Theory of cross-correlation analysis of PIV images. Appl Sci Res 49(3):191-215. https://doi.org/10.1007/ BF00384623

Kordel S, Nowak T, Skoda R, Hussong J (2016) Combined density gradient and velocity field measurements in transient flows by means of Differential Interferometry and Long-range $\mu$ PIV. Exp Fluids 57(9):1-11. https://doi.org/10.1007/s00348-016-2224-7

Koutsiaris AG, Mathioulakis DS, Tsangaris S (1999) Microscope PIV for velocity-field measurement of particle suspensions flowing inside glass capillaries. Meas Sci Technol 10(11):1037-1046. https://doi.org/10.1088/0957-0233/10/11/311

Li M, He Y, Liu Y, Huang C (2018) Hydrodynamic simulation of multi-sized high concentration slurry transport in pipelines. Ocean Eng 163(October 2017):691-705. https://doi.org/10.1016/j.ocean eng.2018.06.046

Lima R, Wada S, Tsubota KI, Yamaguchi T (2006) Confocal micro-PIV measurements of three-dimensional profiles of cell suspension flow in a square microchannel. Meas Sci Technol 17(4):797-808. https://doi.org/10.1088/0957-0233/17/4/026

Lindken R, Merzkirch W (2002) A novel PIV technique for measurements in multiphase flows and its application to two-phase bubbly flows. Exp Fluids 33(6):814-825. https://doi.org/10.1007/ s00348-002-0500-1

Loisel V, Abbas M, Masbernat O, Climent E (2015) Inertia-driven particle migration and mixing in a wall-bounded laminar suspension flow. Phys Fluids 27(12). https://doi.org/10.1063/1.4936402

Meinhart CD, Wereley ST, Santiago JG (2000) A PIV algorithm for estimating time-averaged velocity fields. J Fluids Eng 122(2):285289. https://doi.org/10.1115/1.483256

Olsen MG, Adrian RJ (2000) Out-of-focus effects on particle image visibility and correlation in microscopic particle image velocimetry. Exp Fluids 29(7):S166-S174. https://doi.org/10.1007/s0034 80070018

Raffel M, Willert CE, Wereley S, Kompenhans J (2007) Particle image velocimetry: a practical guide. Experimental Fluid Mechanics. Springer, Berlin Heidelberg

Scharnowski S, Sciacchitano A, Kähler CJ (2018) A new look on the Valid Detection Probability of PIV Vectors. In: LXLASER2018: 19th international symposium on the application of laser and imaging techniques to fluid mechanics

Sciacchitano A (2019) Uncertainty quantification in particle image velocimetry. Meas Sci Technol 30(9). https://doi.org/10.1088/ 1361-6501/ab1db8

Sciacchitano A, Wieneke B (2016) PIV uncertainty propagation. Meas Scie Technol 27(8). https://doi.org/10.1088/0957-0233/27/8/ 084006

Sciacchitano A, Wieneke B, Scarano F (2013) PIV uncertainty quantification by image matching. Meas Sci Technol 24(4). https://doi. org/10.1088/0957-0233/24/4/045302

Sciacchitano A, Neal DR, Smith BL, Warner SO, Vlachos PP, Wieneke B, Scarano F (2015) Collaborative framework for PIV uncertainty quantification: Comparative assessment of methods. Meas Sci Technol 26(7). https://doi.org/10.1088/0957-0233/26/7/074004

Skarman B, Becker J, Wozniak K (1996) Simultaneous 3D-PIV and temperature measurements using a new CCD-based holographic interferometer. https://doi.org/10.1016/0955-5986(96)00006-4 
Smith BL, Oberkampf WL (2014) Limitations of and alternatives to traditional uncertainty quantification for measurements. In: Volume 1D, Symposia: Transport Phenomena in Mixing; Turbulent Flows; Urban Fluid Mechanics; Fluid Dynamic Behavior of Complex Particles; Analysis of Elementary Processes in Dispersed Multiphase Flows; Multiphase Flow With Heat/Mass Transfer in Process Tec, American Society of Mechanical Engineers, pp 1-12. https://doi.org/10.1115/FEDSM2014-22068

Timmins BH, Wilson BW, Smith BL, Vlachos PP (2012) A method for automatic estimation of instantaneous local uncertainty in particle image velocimetry measurements. Exp Fluids 53(4):1133-1147. https://doi.org/10.1007/s00348-012-1341-1

Wang P, Song C, Briscoe C, Makse HA (2008) Particle dynamics and effective temperature of jammed granular matter in a slowly sheared three-dimensional Couette cell. Phys Rev E Stat Nonlinear Soft Matter Phys 77(6):1-15. https://doi.org/10.1103/PhysR evE.77.061309

Westerweel J (1997) Fundamentals of digital particle image velocimetry. Meas Sci Technol 8(12):1379-1392. https://doi.org/10.1088/ 0957-0233/8/12/002

Wiederseiner S, Andreini N, Epely-Chauvin G, Ancey C (2011) Refractive-index and density matching in concentrated particle suspensions: a review. Exp Fluids 50(5):1183-1206. https://doi. org/10.1007/s00348-010-0996-8

Wieneke B (2015) PIV uncertainty quantification from correlation statistics. Meas Sci Technol 26(7):074,002. https://doi.org/10.1088/ 0957-0233/26/7/074002

Willert CE (1996) The fully digital evaluation of photographic PIV recordings. Flow Turbul Combust 56(2-3):79-102

Willert CE, Gharib M (1991) Digital particle image velocimetry. Exp Fluids 10(4):181-193. https://doi.org/10.1007/BF00190388

Xue Z, Charonko JJ, Vlachos PP (2014) Particle image velocimetry correlation signal-to-noise ratio metrics and measurement uncertainty quantification. Meas Sci Technol 25(11). https://doi.org/10. 1088/0957-0233/25/11/115301

Publisher's Note Springer Nature remains neutral with regard to jurisdictional claims in published maps and institutional affiliations. 